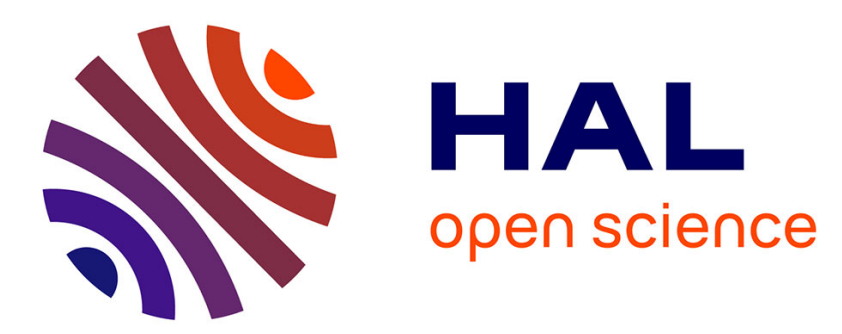

\title{
A phase-field method for computational modeling of interfacial damage interacting with crack propagation in realistic microstructures obtained by microtomography
}

T.T. Nguyen, Julien Yvonnet, Q.-Z Zhu, Michel Bornert, Camille Chateau

\section{- To cite this version:}

T.T. Nguyen, Julien Yvonnet, Q.-Z Zhu, Michel Bornert, Camille Chateau. A phase-field method for computational modeling of interfacial damage interacting with crack propagation in realistic microstructures obtained by microtomography. Computer Methods in Applied Mechanics and Engineering, 2015, 312, pp.567-595. 10.1016/j.cma.2015.10.007 . hal-01213943

\author{
HAL Id: hal-01213943 \\ https://hal.science/hal-01213943
}

Submitted on 9 Oct 2015

HAL is a multi-disciplinary open access archive for the deposit and dissemination of scientific research documents, whether they are published or not. The documents may come from teaching and research institutions in France or abroad, or from public or private research centers.
L'archive ouverte pluridisciplinaire HAL, est destinée au dépôt et à la diffusion de documents scientifiques de niveau recherche, publiés ou non, émanant des établissements d'enseignement et de recherche français ou étrangers, des laboratoires publics ou privés. 


\title{
A phase-field method for computational modeling of interfacial damage interacting with crack propagation in realistic microstructures obtained by microtomography
}

\author{
T.T. Nguyen ${ }^{\mathrm{a}, \mathrm{b}}$, J. Yvonnet ${ }^{\mathrm{a}, *}$, Q.-Z. Zhu ${ }^{\mathrm{a}}$ M. Bornert ${ }^{\mathrm{b}}$, \\ C. Chateau ${ }^{\text {, }}$ \\ ${ }^{a}$ Université Paris-Est, Laboratoire Modélisation et Simulation Multi Échelle \\ MSME UMR 8208 CNRS, 5 bd Descartes, F-77454 Marne-la-Vallée, France. \\ ${ }^{\mathrm{b}}$ Université Paris-Est, Laboratoire Navier, CNRS UMR8205, ENPC, IFSTTAR, 6 \\ et 8 avenue Blaise Pascal, 77455 Marne-la-Vallée Cedex, France.
}

\begin{abstract}
In this work, a formulation is developed within the phase field method for modeling interactions between interfacial damage and bulk brittle cracking in complex microstructures. The method is dedicated to voxel-based models of highly complex microstructures, as obtained from X-ray microtomography images. A smoothed displacement jump approximation is introduced by means of level-set functions to overcome the issue of pixelized interfaces in voxel-based models. A simple technique is proposed to construct the level-set function in that case. Compared to recent work aiming at modeling cohesive cracks within the phase field method, our framework differs in several points: the formulation is such that interfaces are not initially damaged; no additional variables are required to describe the discontinuities at the interface and fatigue cracks can be modeled. The technique allows interaction between bulk and interface cracks, e.g. nucleation from interfaces and propagation within the matrix, and for arbitrary geometries and interactions between cracks. Several benchmarks are presented to validate the model. The technique is illustrated through numerical examples involving complex microcracking in X-ray CT image-based models of concrete microstructures.
\end{abstract}

Key words: Cracks, Phase field, Highly heterogenous materials, Interfacial damage, Voxel-based models, Microcracking 


\section{Introduction}

Microtomography or X-ray CT-scan experimental setups [33] have been democratized in the recent years and realistic models of microstructures obtained from 3D imaging techniques can now be routinely generated for many materials at various scales. Furthermore, it is now possible to use these models in numerical simulations to evaluate mechanical and other physical properties of complex materials like bones [34,11], concrete [43,30], coke blend [36], filled elastomers [1], among many others.

Beyond evaluating linear properties, a major objective is to predict the strength and failure of materials by simulating damage and microcracking at the microscopic level in X-ray CT scan voxel models. In complex materials like concrete or cementitious materials, both matrix cracking and interfacial damage can occur and interact. For example, it has been suggested that the strength of concrete is largely attributed to the properties of mortar-aggregate interfaces $[16,19]$. The interaction of an interphase on a matrix crack has been studied in ceramic matrix composites [24]. Damage by fibre cracking or decohesion in metallic composites reinforced by brittle fibres has been studied in [37].

Many numerical methods have been developed to investigate interface damage, including thin interphases with graded properties [40], or cohesive zone models. Because of its versatility, cohesive zone modeling (CZM) is one of the primary method to handle discrete crack propagation in diverse types of materials. This concept was introduced by Barenblatt [4] and Dugdale [14] to address the stress singularity at a crack tip. In these models, all nonlinearities take place in a cohesive zone ahead of the main crack tip, which is associated with the physical fracture process zone of the material. The cohesive laws have been embedded into finite element analysis in Needleman [25] and Tvergaard and Hutchinson [38] or have been modeled by cohesive finite elements like in Camacho and Ortiz [8], Xu and Needleman [42], and Ortiz and Pandolfi [28], or in a dynamic context by Zhou and Molinari in [44]. An overview of cohesive elements techniques can be found in Chandra et al [10].

Simulating interfacial damage and its interaction with matrix cracking in voxel-based models of complex microstructures of real materials is highly challenging. Indeed, in voxel models consisting of regular meshes, the interfaces are not explicitly described and normal vectors are not defined. Furthermore, due to the highly heterogeneous nature of real microstructures like concrete, a very complex network of cracks can nucleate, propagate and interact, either from the interfaces and then through the solid phases, or the opposite. Simulating such complex networks of cracks is a well-known issue for meshing

* Correspondance to J. Yvonnet

Email address: julien.yvonnet@univ-paris-est.fr (J. Yvonnet ). 
algorithms and remeshing techniques are not suited to regular meshes based on voxels, where maintaining the same mesh during the simulation is favorable for robustness and parallel computing purpose.

Apart from cohesive elements, the eXtended Finite Element Method (XFEM) $[22,35]$ can alleviate the issue of describing arbitrary cracks in regular meshes by using an enriched FEM discretization scheme with additional nodal variables for describing displacement jump over arbitrary surfaces not matching the mesh. However, this technique has shown difficulties to describe crack nucleation and requires level-set function construction to describe the crack, which can be cumbersome when multiple cracks interact. We also mention a related method, called Thick Level-Set method (TLS) $[5,9]$ in which a level-set function is employed to separate the undamaged zone from the damaged one, and where the crack is a consequence of the damage front motion, allowing crack initiation.

More recently, the Phase Field Method has been introduced, based on the pioneer works of Marigo and Francfort [15]. This technique makes use of a regularized description of discontinuities through an additional phase field variable and strongly alleviates meshing problems for describing brittle cracking. Furthermore, the phase field problem being solved at each increment in an algorithmic framework [20], nucleation, interaction and arbitrary crack morphologies can be handled in regular meshes. The technique has proved to be very well suited to the simulation of microcracking in complex voxel-based models of concrete microstructures in [27]. The technique has been recently adapted to cohesive cracks in [39].

In the present work, we propose a new phase field formulation taking into account both bulk brittle fracture and interfacial damage. The present method is different from the work of Verhoosel and de Borst [39] by the following points. First, we introduce a new energetic formulation mixing bulk damageable energy and cohesive surface energy such that the interfaces do not initially involve discontinuities and thus no damage in the phase field sense. In this formulation, the phase field describes the bulk crack surface density, as well as the interface crack density, allowing interaction between both crack types in a simple manner. Second, we have investigated two models, one involving internal variables to describe interfacial damage within cohesive traction law, and the other without internal variable. We show that in our formulation, the phase field is sufficient to model crack opening and re-closure without internal variables for interfaces. Third, to describe the diffuse displacement jump at the interface, we use a level-set method without additional variables, unlike in [39]. A special algorithm dedicated to the construction of the level-set functions in voxel-based models of complex microstructures is introduced. The features of the proposed method are summarized as follows: 
- The technique allows simulating interfacial cracking in voxel-based regular FEM models of real microstructures, and their interaction with matrix cracks.

- No additional variables are needed to describe the jump at the interface.

- The solution is convergent with respect to the mesh and the crack path is mesh-independent.

- The phase field describes both the crack density in the matrix and the interface crack density; it can be used as an internal variable to model irreversible damage of the interface.

The overview of the paper is as follows. In section 2 the diffuse approximation of discontinuities related to cracks and interfaces using phase field is introduced. In section 3, we propose a modified phase field framework able to describe both bulk microcracking and interface damage. In section 4 , a simple method is presented to construct the level-set function from voxelbased models in regular meshes. In section 5, the numerical details and FEM discretization details are presented. Finally, the method is validated and illustrated by interfacial damage benchmarks and practical examples involving microcracking in voxel-based models of microstructures in section 6 .

\section{Diffuse approximation of discontinuous fields}

\subsection{Smeared approximation of cracks and interfaces}

Let $\Omega \in \mathbb{R}^{d}$ be an open domain describing a solid with external boundary $\partial \Omega$. The solid is heterogeneous, and contains internal interfaces between the phases, collectively denoted by $\Gamma^{I}$. During the loading, cracks may propagate in the solid phases and can pass through the interfaces as depicted in Fig. 1 (a). The crack surfaces are collectively denoted by $\Gamma$. In the present work, we adopt the regularized framework proposed in $[20,23,2]$ for replacing cracks and interfaces surfaces by regularized approximation functions. Cracks are associated with an evoluting phase field $d(\mathbf{x}, t)$ (see Fig 1 (c)), while interfaces between phases are associated with a fixed scalar field $\beta(\mathbf{x})$ (see Fig 1 (b)). The phase field $d(\mathbf{x}, t)$ satisfies the following equations (see [20] for more details):

$$
\left\{\begin{array}{l}
d(\mathbf{x}, t)-l_{d}^{2} \triangle d(\mathbf{x}, t)=0 \text { in } \Omega \\
d(\mathbf{x}, t)=1 \text { on } \Gamma \\
\nabla d(\mathbf{x}, t) \cdot \mathbf{n}=0 \text { on } \partial \Omega
\end{array}\right.
$$

where $\Delta($.$) is the Laplacian, l_{d}$ is a regularization parameter describing the actual width of the smeared crack, and $\mathbf{n}$ the outward normal to $\partial \Omega$. Note 


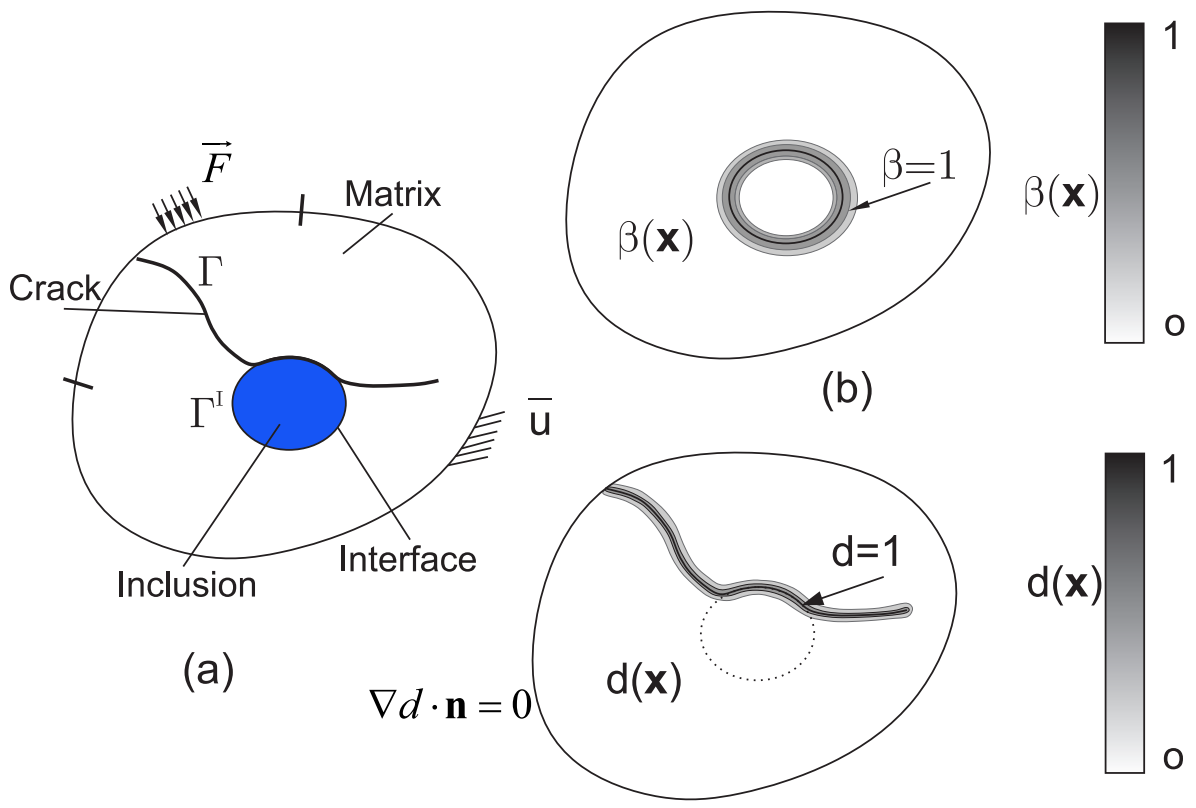

(c)

Fig. 1. Regularized representation of a crack and smeared crack: (a) a body containing an interface and a crack possibly passing through the interface; (b) smeared representation of the interface; (c) smeared representation of the crack.

that $l_{d}$ is a length whatever the dimension of the space of analysis is. In addition, even though the vocabulary is sometimes specific to $2 \mathrm{D}$ problems, the framework is fully valid for three-dimensional cases. In the following, we omit the dependence to time for the sake of conciseness, assuming that the problem is solved at each load increment $t_{n}, n=1, \ldots, N$, with $N$ being the number of load increments. It can be shown (see e.g. [20]) that (1) is the Euler-Lagrange equation associated with the variational problem:

$$
d(\mathbf{x}, t)=\operatorname{Arg}\left\{\inf _{d \in \mathcal{S}_{d}} \Gamma^{d}(d)\right\}
$$

where $\mathcal{S}_{d}=\{d \mid d(\mathbf{x})=1 \forall \mathbf{x} \in \Gamma\}$ and

$$
\Gamma^{d}(d)=\int_{\Omega} \gamma_{d}(d) d \Omega
$$

where $\Gamma^{d}(d)$ represents the total crack length, i.e. the total length (or area in $3 \mathrm{D})$ of crack per unit area in the 2D case, and total surface of crack, per unit volume in the $3 \mathrm{D}$ case. In $(3) \gamma_{d}(d)$ has the unit of the inverse of a length and is defined by:

$$
\gamma_{d}(d(\mathbf{x}))=\frac{1}{2 l_{d}} d(\mathbf{x})^{2}+\frac{l_{d}}{2} \nabla d(\mathbf{x}) \cdot \nabla d(\mathbf{x})
$$


For $l_{d} \rightarrow 0$ the above variational principle leads to the exact description of the sharp crack topology $\Gamma$. The origin of the definition (4) can be found e.g. in [7] or [21]. First, a model of regularization is chosen to replace the sharp damage gradient near the crack. The corresponding differential equations are then integrated by means of a Galerkin-type weak form. These equations are the Euler-Lagrange equations of a variational principle, in which the total crack length to be minimized and the crack density function can be identified (see more details in [21]). Note that other choices of regularization are possible. For example, in [6], smoother regularization schemes have been investigated, leading to a different expression for $\gamma(d)$.

The interfaces are here described in the same manner. The field $\beta(\mathbf{x})$ satisfies:

$$
\left\{\begin{array}{l}
\beta(\mathbf{x})-l_{\beta}^{2}(\mathbf{x}) \triangle \beta(\mathbf{x})=0 \text { in } \Omega \\
\beta(\mathbf{x})=1 \text { on } \Gamma^{I} \\
\nabla \beta(\mathbf{x}) \cdot \mathbf{n}=0 \text { on } \partial \Omega
\end{array}\right.
$$

where $l_{\beta}$ is the regularization parameter describing the width of the regularized interfaces. Similarly, (5) is the Euler-Lagrange equation associated with the variational problem:

$$
\beta(\mathbf{x})=\operatorname{Arg}\left\{\inf _{\beta \in \mathcal{S}_{\beta}} \Gamma_{\beta}(\beta)\right\},
$$

where $\mathcal{S}_{\beta}=\left\{\beta \mid \beta(\mathbf{x})=1 \forall \mathbf{x} \in \Gamma^{I}\right\}$ and $\Gamma_{\beta}(\beta)=\int_{\Omega} \gamma_{\beta}(\beta) d \Omega$, where $\Gamma_{\beta}$ represents the total interface length and $\gamma_{\beta}$ is defined by

$$
\gamma_{\beta}(\beta)=\frac{1}{2 l_{\beta}} \beta(\mathbf{x})^{2}+\frac{l_{\beta}}{2} \nabla \beta(\mathbf{x}) \cdot \nabla \beta(\mathbf{x})
$$

For $l_{\beta} \rightarrow 0$ the above variational principle leads to the exact description of the sharp interface $\Gamma^{I}$. In the following, we will choose identical regularization lengths for cracks and interfaces, i.e. $l_{d}=l_{\beta}=l$ and that $\beta(\mathbf{x})$ does not change throughout the simulation (the interfaces do not evolve), unless specified.

\subsection{Smeared displacement jump approximation}

In the present work, the displacement jump $\llbracket \mathbf{u}(\mathbf{x}) \rrbracket$ created by interface decohesion is approximated as a smooth transition, and defined as follows. Let $\Gamma^{I}$ be the interface. We define $\Gamma^{I}$ as the zero level-set of a function $\phi(\mathbf{x})$, such 


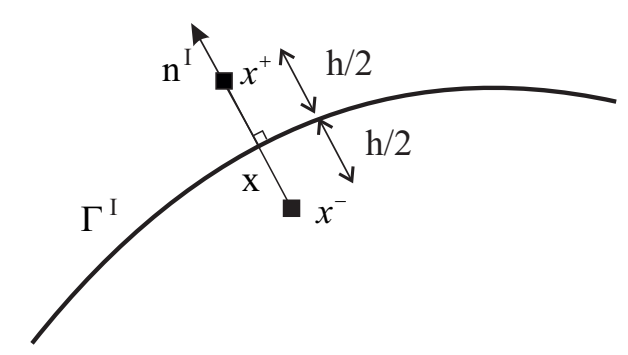

Fig. 2. Approximation of the displacement jump across the interface.

that:

$$
\left\{\begin{array}{l}
\phi(\mathbf{x})>0 \text { for } \mathrm{x} \in \Omega^{\mathrm{i}} \\
\phi(\mathbf{x})<0 \text { for } \mathrm{x} \in \Omega / \Omega^{\mathrm{i}} \\
\phi(\mathbf{x})=0 \text { for } \mathrm{x} \in \Gamma^{\mathrm{I}}
\end{array}\right.
$$

where $\Omega^{i}$ denotes the set of inclusions, and $\Omega / \Omega^{i}$ the matrix ${ }^{1}$. Let $h$ be a small scalar parameter, $\mathbf{x} \in \Gamma^{I}$ and $\mathbf{n}^{I}$ the normal vector to $\Gamma^{I}$ at the point $\mathbf{x}$. If the function $\phi(\mathbf{x})$ is known, the normal vector to $\Gamma^{I}$ is found through:

$$
\mathbf{n}^{I}(\mathbf{x})=\frac{\nabla \phi(\mathbf{x})}{\|\nabla \phi(\mathbf{x})\|}
$$

Using a Taylor expansion at first order of the assumed smoothed regularized displacement fields $\mathbf{u}(\mathbf{x})$, we can express (see Fig. 2):

$$
\begin{aligned}
& \mathbf{u}\left(\mathbf{x}+\frac{h}{2} \mathbf{n}^{I}\right) \simeq \mathbf{u}(\mathbf{x})+\frac{h}{2} \nabla \mathbf{u}(\mathbf{x}) \mathbf{n}^{I} \\
& \mathbf{u}\left(\mathbf{x}-\frac{h}{2} \mathbf{n}^{I}\right) \simeq \mathbf{u}(\mathbf{x})-\frac{h}{2} \nabla \mathbf{u}(\mathbf{x}) \mathbf{n}^{I}
\end{aligned}
$$

Then in a regularized context, the displacement jump is not only defined on the interface but over all the domain, and its expression at any point $\mathbf{x} \in \Omega$ is given by:

$$
\begin{aligned}
& \llbracket \mathbf{u}(\mathbf{x}) \rrbracket \simeq \mathbf{w}(\mathbf{x})=\mathbf{u}\left(\mathbf{x}+\frac{h}{2} \mathbf{n}^{I}\right)-\mathbf{u}\left(\mathbf{x}-\frac{h}{2} \mathbf{n}^{I}\right) \\
& =h \nabla \mathbf{u}(\mathbf{x}) \frac{\nabla \phi(\mathbf{x})}{\|\nabla \phi(\mathbf{x})\|} .
\end{aligned}
$$

\footnotetext{
1 Note that even though presented here for bi-material interfaces, $P$ interfaces could be considered if $P$ couples of nodes with different materials are involved. In that cse, the $P$ interfaces would each have their own geometrical definition through $P$ fields $\beta_{i}(\mathbf{x}), i=1, \ldots, P$ and $P$ coefficients $g_{c}^{i}, i=1, \ldots, P$.
} 
where $\mathbf{w}(\mathbf{x})$ denotes the smoothed displacement jump approximation.

A detailed description for the numerical computation of $\phi(\mathbf{x})$ for an arbitrary morphology of microstructure described by a regular grid of voxels is provided in section 4 .

\section{Phase field incorporating bulk brittle fracture and cohesive in- terfaces}

\subsection{Energy functional}

Let us consider a two-phase solid as described in section 2.1. The solid contains both cracks and interfaces, implying strong displacement discontinuities. In a standard framework of sharp discontinuity description, the total energy is given by:

$$
E=\int_{\Omega} W_{u}(\varepsilon(\mathbf{u})) d \Omega+\int_{\Gamma} g_{c} d \Gamma+\int_{\Gamma^{I}} \Psi^{I}(\llbracket \mathbf{u} \rrbracket, \boldsymbol{\alpha}) d \Gamma
$$

In (13) $g_{c}$ is the toughness, $\Psi^{I}$ is a strain density function depending on the displacement jump across the interface $\Gamma^{I}$ and $\boldsymbol{\alpha}$ is a history parameter. If a regularized description for strong discontinuities related to both cracks and interfaces is adopted (substituting $\llbracket \mathbf{u} \rrbracket$ by $\mathbf{w}(\mathbf{x})$ ), then propose to split the infinitesimal strain tensor into a part related to the bulk and a part induced by the smoothed jump at the interfaces, denoted by $\varepsilon^{e}$ and $\tilde{\varepsilon}$, respectively:

$$
\varepsilon=\varepsilon^{e}+\tilde{\varepsilon} .
$$

This decomposition is proposed here such that $\tilde{\varepsilon} \rightarrow 0$ away from the interfaces, i.e. when $\beta(\mathbf{x}) \rightarrow 0$. Then, we propose to replace the above functional by the following one:

$$
\begin{aligned}
& E=\int_{\Omega} W_{u}^{e}\left(\varepsilon^{e}(\mathbf{u}, \beta), d\right) d \Omega+\int_{\Omega}[1-\beta(\mathbf{x})] g_{c} \gamma_{d}(d) d \Omega \\
& +\int_{\Omega} \Psi^{I}(\mathbf{w}, \boldsymbol{\alpha}) \gamma_{\beta}(\beta) d \Omega .
\end{aligned}
$$

where $\gamma_{d}$ and $\gamma_{\beta}$ have been defined in section (2.1).

The factor $[1-\beta(\mathbf{x})]$ is introduced so as to verify that for $\beta(\mathbf{x}) \rightarrow 0$ (away from the interface) which includes $\gamma_{\beta} \rightarrow 0$ and $\varepsilon^{e} \rightarrow \varepsilon$. As a consequence of these definitions, we recover the regularized energy functional for brittle 
fracture without interfaces $[15,20]$ :

$$
E=\int_{\Omega} W_{u}(\varepsilon(\mathbf{u}), d) d \Omega+\int_{\Omega} g_{c} \gamma_{d}(d) d \Omega
$$

In (15), we identify

$$
W=W_{u}^{e}\left(\varepsilon^{e}(\mathbf{u}, \beta), d\right)+[1-\beta(\mathbf{x})] g_{c} \gamma_{d}(d)+\Psi^{I}(\mathbf{w}, \boldsymbol{\alpha}) \gamma_{\beta}(\beta)
$$

as the free energy. Using the variational principle for minimizing $E$ with respect to displacements, i.e.

$$
\mathbf{u}(\mathbf{x})=\operatorname{Arg}\left\{\inf _{\mathbf{u} \in \mathcal{S}_{u}}\left(E(\mathbf{u}, d, \beta, \alpha)-W^{e x t}\right)\right\}
$$

where $\mathcal{S}_{u}=\left\{\mathbf{u} \mid \mathbf{u}(\mathbf{x})=\overline{\mathbf{u}}\right.$ on $\left.\partial \Omega_{u}, \quad \mathbf{u} \in H^{1}(\Omega)\right\}$ and $W^{\text {ext }}=\int_{\Omega} \mathbf{f} \cdot \mathbf{u} d \Omega+\int_{\partial \Omega_{F}} \overline{\mathbf{F}}$. $\mathbf{u} d \Gamma$ with $\mathbf{f}$ and $\overline{\mathbf{F}}$ being body forces and prescribed traction over the boundary $\partial \Omega_{F}$, we obtain the weak form for $\mathbf{u}(\mathbf{x}) \in \mathcal{S}_{u}$ :

$$
\begin{aligned}
& \int_{\Omega} \frac{\partial W_{u}^{e}}{\partial \varepsilon^{e}}: \varepsilon^{e}(\delta \mathbf{u}) d \Omega+\int_{\Omega} \frac{\partial \Psi^{I}(\mathbf{w}, \boldsymbol{\alpha})}{\partial \mathbf{w}} \cdot \delta \mathbf{w} \gamma_{\beta}(\beta) d \Omega \\
& =\int_{\Omega} \mathbf{f} \cdot \delta \mathbf{u} d \Omega+\int_{\partial \Omega_{F}} \overline{\mathbf{F}} \cdot \delta \mathbf{u} d \Gamma=\delta W^{e x t}
\end{aligned}
$$

In the absence of body forces $(\mathbf{f}=\mathbf{0})$, invoking the local balance of stress and global balance of power (i.e. $\int_{\Omega} \boldsymbol{\sigma}^{e}: \boldsymbol{\varepsilon}^{e} d \Omega=\delta W^{e x t}$ ) and using the divergence theorem we can re-write (19) as:

$$
\int_{\Omega} \boldsymbol{\sigma}^{e}: \boldsymbol{\varepsilon}^{e}(\delta \mathbf{u}) d \Omega+\mathbf{t}(\mathbf{w}, \boldsymbol{\alpha}) \cdot \delta \mathbf{w} \gamma_{\beta}(\beta) d \Omega-\int_{\Omega} \boldsymbol{\sigma}^{e}: \nabla^{s} \delta \mathbf{u} d \Omega=0
$$

where $\boldsymbol{\sigma}^{e}=\frac{\partial W_{e}}{\partial \boldsymbol{\varepsilon}^{e}}$ is the Cauchy stress and $\mathbf{t}(\mathbf{w}, \boldsymbol{\alpha})=\frac{\partial \Psi^{I}(\mathbf{w}, \boldsymbol{\alpha})}{\partial \mathbf{w}}$ is the traction vector acting on the interface oriented by $\mathbf{n}^{I}$ and associated with the displacement jump at the interface, as a consequence of the assumptions on the elastic behaviour of the bulk material and the partitioning of the local strain near the interface. In (20), $\delta \mathbf{w}$ is obtained using (12) as:

$$
\delta \mathbf{w}(\mathbf{x})=h \nabla \delta \mathbf{u}(\mathbf{x}) \frac{\nabla \phi(\mathbf{x})}{\|\nabla \phi(\mathbf{x})\|}
$$

Using $\boldsymbol{\sigma}^{e} \mathbf{n}=\mathbf{t}$, Eq. (20) can be further re-written as:

$$
\int_{\Omega} \boldsymbol{\sigma}^{e}:\left\{\boldsymbol{\varepsilon}^{e}(\delta \mathbf{u})+\mathbf{n} \otimes \delta \mathbf{w} \gamma_{\beta}(\beta)-\nabla^{s} \delta \mathbf{u}\right\} d \Omega=0
$$

which is satisfied for an admissible strain field in the form:

$$
\varepsilon^{e}=\nabla^{s} \mathbf{u}-\mathbf{n} \otimes^{s} \mathbf{w} \gamma_{\beta}
$$


where $\left(\nabla^{s} \mathbf{u}\right)_{i j}=\frac{1}{2}\left(u_{i, j}+u_{j, i}\right)$ and $\left(\mathbf{n} \otimes^{s} \mathbf{w}\right)_{i j}=\frac{1}{2}\left(n_{i} w_{j}+w_{i} n_{j}\right)$. From 14 we identify $\tilde{\varepsilon}$ as:

$$
\tilde{\varepsilon}=\mathbf{n} \otimes^{s} \mathbf{w} \gamma_{\beta} .
$$

\subsection{Phase field problem}

The reduced Clausius-Duhem inequality relative to the evolution of the damage parameter $d$ is defined as

$$
\mathcal{A} \dot{d} \geq 0
$$

where $\mathcal{A}=-\frac{\partial W}{\partial d}$ is the thermodynamic force associated with $d$. Assuming a threshold function $F(\mathcal{A})$ such that no damage occurs below that value in the form:

$$
F(\mathcal{A})=\mathcal{A} \leq 0
$$

and invoking the principle of maximum dissipation, the dissipation $\mathcal{A} \dot{d}$ must be maximum under the constraint (26). It yields that for $\dot{d}>0, F=0$ which gives (see more details in [27]):

$$
F=-\frac{\partial W}{\partial d}=-\left\{\frac{\partial W_{u}^{e}}{\partial d}+[1-\beta] g_{c} \delta \gamma_{d}(d)\right\}=0
$$

where [20]:

$$
\delta \gamma_{d}(d)=\frac{d}{l_{d}}-l_{d} \Delta d
$$

We assumed an isotropic elastic behavior of the phases in both the initial and damaged state, with initial Lamé's coefficients $\lambda$ and $\mu$. To take into account unilateral contact, damage is assumed to modify the sole tensile part of the elastic energy, which is defined as

$$
W_{u}^{e}=\Psi_{e}^{+}\left(\varepsilon^{e}\right)\{g(d)+k\}+\Psi_{e}^{-}\left(\varepsilon^{e}\right)
$$

where

$$
\varepsilon^{e}=\varepsilon^{e+}+\varepsilon^{e-}
$$

and

$$
\Psi_{e}^{ \pm}(\varepsilon)=\frac{\lambda}{2}\left(\left\langle\operatorname{Tr}\left(\varepsilon^{e}\right)\right\rangle_{ \pm}\right)^{2}+\mu \operatorname{Tr}\left\{\left(\varepsilon^{e \pm}\right)^{2}\right\} .
$$


These two contributions to the strain energy are then defined as:

$$
\boldsymbol{\varepsilon}^{e \pm}=\sum_{i=1}^{D}\left\langle\varepsilon^{i}\right\rangle_{ \pm} \mathbf{n}^{i} \otimes \mathbf{n}^{i}
$$

and $\varepsilon^{i}$ and $\mathbf{n}^{i}$ are the eigenvalues and eigenvectors of $\varepsilon^{e}$, i.e. satisfying $\varepsilon^{e} \mathbf{n}^{i}=$ $\varepsilon^{i} \mathbf{n}^{i}$. In (31) - (32), $\langle x\rangle_{ \pm}=(x \pm|x|) / 2$. The degradation function $g(d)$ is assumed to have the simple form $g(d)=(1-d)^{2}$. The small parameter $k<<1$ is introduced to maintain the well-posedness of the system for partially broken parts of the domain while perturbating the strain energy in non damaged parts to a negligible level. It follows that when $\dot{d}>0$ then:

$$
-2(1-d) \Psi_{e}^{+}+[1-\beta] g_{c} \delta \gamma_{d}(d)=0
$$

It is worth noting that as $2(1-d) \Psi_{e}^{+} \geq 0$, then $\delta \gamma(d) \geq 0$, yielding $\dot{\Gamma}^{d} \geq 0$. To handle loading and unloading history, we follow Miehe et al. [20] and introduce the strain history function

$$
\mathcal{H}(\mathbf{x}, t)=\max _{\tau \in[0, t]}\left\{\Psi_{e}^{+}(\mathbf{x}, \tau)\right\}
$$

which is substituted to $\Psi_{e}^{+}$in (34). It yields the following phase field problem to be solved to evaluate the field $d(\mathbf{x}, t)$ at time $t$, using (28):

$$
\left\{\begin{array}{l}
2(1-d) \mathcal{H}-(1-\beta) \frac{g_{c}}{l_{d}}\left\{d-l_{d}^{2} \Delta d\right\}=0 \text { in } \Omega \\
d(\mathbf{x})=1 \text { on } \Gamma \\
\nabla d(\mathbf{x}) \cdot \mathbf{n}=0 \text { on } \partial \Omega .
\end{array}\right.
$$

The associated weak form is obtained as:

$$
\int_{\Omega}\left\{\left(2 \mathcal{H}+[1-\beta] \frac{g_{c}}{l_{d}}\right) d \delta d+[1-\beta] g_{c} l_{d} \nabla d \cdot \nabla(\delta d)\right\} d \Omega=\int_{\Omega} 2 \mathcal{H} \delta d d \Omega .(36
$$

Remark The choice of $l$ has been discussed in [3]. In the mentioned work, a relationship has been established between $l$ and material parameters. For example, assuming uniaxial traction of a bar, the relationship:

$$
\sigma_{c}^{+}=\frac{3}{16} \sqrt{\frac{3}{2}} \sqrt{\frac{E g_{c}}{l}}
$$

where $\sigma_{c}^{+}$is the critical value of stress in traction. As mentioned in [3], the shape of the damaged zone (and hence the approximate crack path) can significantly change with the length $l$ if $l$ is not sufficiently small with respect to the size $L$ of the domain $\Omega$. The authors then define two cases: (a) $l$ is considered as a pure numerical parameter of the regularized model of brittle fracture; (b) $l$ is seen as a real material parameter for a gradient damage model. In the first case, it is recommended to take $l$ as small as possible to better approximate brittle fracture, with regards to the size of 
the mesh. In the second case, $l$ should be identified by experimental data through relationships like (37). Such analysis is presently under study with experimental validations in [26].

\subsection{Displacement problem}

\subsubsection{Governing equations}

The weak form associated with the displacement problem has been defined in (19), with the above described strain energy, the Cauchy stress now reads:

$$
\begin{aligned}
& \boldsymbol{\sigma}^{e}=\frac{\partial \Psi_{e}^{+}}{\partial \varepsilon^{e}}\{g(d)+k\}+\frac{\partial \Psi_{e}^{-}}{\partial \varepsilon^{e}} \\
& =\left((1-d)^{2}+k\right)\left\{\lambda\left\langle\operatorname{Tr} \varepsilon^{e}\right\rangle_{+} \mathbf{1}+2 \mu \varepsilon^{e+}\right\}+\lambda\left\langle\operatorname{Tr} \varepsilon^{e}\right\rangle_{-} \mathbf{1}+2 \mu \varepsilon^{e-} .
\end{aligned}
$$

In the applications of the following work, we have used the numerical value $k=10^{-8}$.

\subsubsection{Models for interface damage}

Regarding the constitutive relation of the interface, two models are investigated in the present paper. The general form in $2 \mathrm{D}$ is given by

$$
\mathbf{t}(\mathbf{w}, \boldsymbol{\alpha})=\left[t^{n}, t^{t}\right]^{\mathrm{T}}
$$

where $t^{n}$ and $t^{t}$ denote normal and tangential parts of the traction vector $\mathbf{t}$ across the interface $\Gamma^{I}$ oriented by its normal $\mathbf{n}^{I}$. In a first model (called M1), a nonlinear elastic cohesive model without dependence on history is used $[13,39]$ :

$$
t^{n}=g_{c}^{I} \frac{\mathrm{W}^{\mathrm{n}}}{\delta^{n}} \exp \left(-\frac{\mathrm{w}^{\mathrm{n}}}{\delta^{\mathrm{n}}}\right) \exp \left(-\frac{\left(\mathrm{w}^{\mathrm{t}}\right)^{2}}{\left(\delta^{\mathrm{t}}\right)^{2}}\right),
$$

where $\mathrm{w}^{\mathrm{n}}=\mathbf{w} \cdot \mathbf{n}^{\mathrm{I}}$ and $\mathrm{w}^{\mathrm{t}}=\mathbf{w} \cdot \mathbf{m}^{\mathrm{I}}, \mathbf{m}^{I}$ being a tangent vector to $\Gamma^{I}, g_{c}^{I}$ is the toughness associated with the interface, and

$$
t^{t}=2 g_{c}^{I} \frac{\mathrm{w}^{\mathrm{t}}}{\delta^{t}}\left(1+\frac{\mathrm{w}^{\mathrm{n}}}{\delta^{n}}\right) \exp \left(-\frac{\mathrm{w}^{\mathrm{n}}}{\delta^{\mathrm{n}}}\right) \exp \left(-\frac{\left(\mathrm{w}^{\mathrm{t}}\right)^{2}}{\left(\delta^{\mathrm{t}}\right)^{2}}\right) .
$$

The relation between $\delta^{n}$, the toughness $g_{c}^{I}$ (which corresponds to the total area under the traction-opening curve) and the fracture strength $t_{u}$ is given by $\delta^{n}=g_{c}^{I} /\left(t_{u} e\right), e=\exp (1)$ (see Fig. 3). 


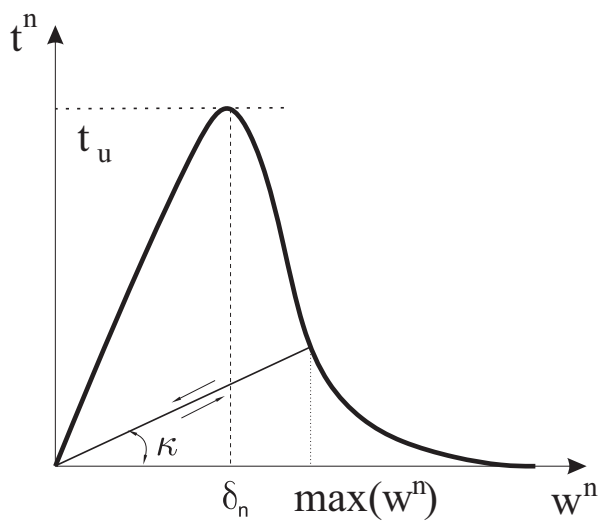

Fig. 3. Cohesive model for the interfaces.

Another possible model, denoted by (M2), introduces a history parameter to describe the irreversible damage in the interface (see Fig. 3). In that case, the expression of the traction at the interface is given e.g. for the normal traction by

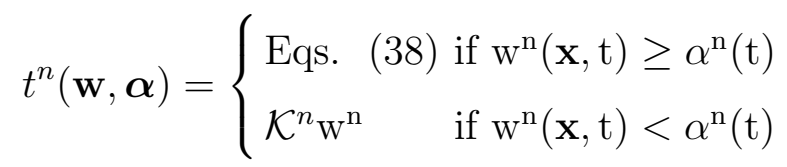

with:

$$
\alpha^{n}(\mathbf{x}, t)=\max _{\tau \in[0, t]}\left\{\mathrm{w}^{\mathrm{n}}(\mathbf{x}, \tau)\right\}
$$

and

$$
\mathcal{K}^{n}=\frac{t^{n}(\alpha(\mathbf{x}, \mathrm{t}))}{\alpha^{n}(\mathbf{x}, \mathrm{t})}
$$

Similarly,

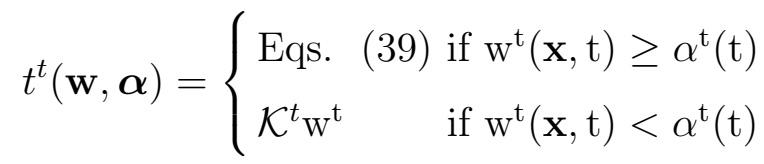

with:

$$
\alpha^{t}(\mathbf{x}, t)=\max _{\tau \in[0, t]}\left\{\mathrm{w}^{\mathrm{t}}(\mathbf{x}, \tau)\right\},
$$

and

$$
\mathcal{K}^{t}=\frac{t^{t}\left(\alpha^{t}(\mathbf{x}, \mathrm{t})\right)}{\alpha^{t}(\mathbf{x}, \mathrm{t})}
$$


Note that in the model M1, no internal variable is involved. In the model M2, we introduce only one internal variable $\alpha^{n}$ for the normal traction component in the cohesive model for the sake of simplicity, the tangential part of the traction was assumed to be zero.

\subsection{Linearization of displacement problem}

We note that for a fixed value of $d$, the mechanical problem (19) is nonlinear, because of the decomposition (30) which requires computing the eigenvalues of $\varepsilon^{e}$ and the nonlinear interfacial law (40)-(41). In what follows, we introduce a linearization procedure to solve the problem by the Newton method.

From (19) and (38) can we rewrite the balance equation as

$$
\begin{aligned}
& \mathcal{R}=\int_{\Omega} \boldsymbol{\sigma}^{e}: \boldsymbol{\varepsilon}^{e}(\delta \mathbf{u}) d \Omega+\int_{\Omega} \gamma_{\beta}(\mathbf{x}) \mathbf{t}(\mathbf{w}, \alpha) \cdot \delta \mathbf{w} d \Omega \\
& -\int_{\Omega} \mathbf{f} \cdot \delta \mathbf{u} d \Omega-\int_{\partial \Omega_{F}} \overline{\mathbf{F}} \cdot \delta \mathbf{u} d \Gamma=0
\end{aligned}
$$

where $\varepsilon^{e}(\delta \mathbf{u})=\nabla^{s}(\delta \mathbf{u})-\mathbf{n} \otimes^{s} \delta \mathbf{w} \gamma_{\beta}$. In a standard Newton method, the displacements are updated for each loading increment by solving the tangent problem:

$$
D_{\Delta u} \mathcal{R}\left(\mathbf{u}^{k}, d\right)=-\mathcal{R}\left(\mathbf{u}^{k}, d\right)=0
$$

where $\mathbf{u}^{k}$ is the displacement solution known from the previous iteration. The displacement corrections are obtained as

$$
\mathbf{u}^{k+1}=\mathbf{u}^{k}+\Delta \mathbf{u}
$$

In (49),

$$
D_{\Delta \mathbf{u}} \mathcal{R}\left(\mathbf{u}^{k}\right)=\int_{\Omega} \frac{\partial \boldsymbol{\sigma}^{e}}{\partial \boldsymbol{\varepsilon}^{e}}: \boldsymbol{\varepsilon}^{e}(\Delta \boldsymbol{\varepsilon}): \boldsymbol{\varepsilon}^{e}(\delta \boldsymbol{\varepsilon})+\int_{\Omega} \frac{\partial \mathbf{t}(\mathbf{w})}{\partial \mathbf{w}}: \Delta \mathbf{w}: \delta \mathbf{w} d \Omega
$$

with

$$
\Delta \mathbf{w}(\mathbf{x})=h \nabla \Delta \mathbf{u}(\mathbf{x}) \frac{\nabla \phi(\mathbf{x})}{\|\nabla \phi(\mathbf{x})\|}
$$

and

$$
\frac{\partial \boldsymbol{\sigma}^{e}}{\partial \boldsymbol{\varepsilon}^{e}}=\mathbb{C}(\mathbf{u}, d)
$$




$$
=\left((1-d)^{2}+k\right)\left\{\lambda R^{+}[\mathbf{1}]^{T}[\mathbf{1}]+2 \mu \mathbf{P}^{+}\right\}+\left\{\lambda R^{-}[\mathbf{1}]^{T}[\mathbf{1}]+2 \mu \mathbf{P}^{-}\right\}
$$

where the operators $R^{ \pm}$and $\mathbf{P}^{ \pm}$have been defined in [27], and $\lambda, \mu$ are the material parameters.

For the model M1, we obtain:

$$
\frac{\partial \mathbf{t}(\mathbf{w})}{\partial \mathbf{w}}=\mathbf{K}_{I}=\left[\begin{array}{cc}
D_{n n} & D_{n t} \\
D_{t n} & D_{t t}
\end{array}\right]
$$

with

$$
D_{n n}=\frac{\partial \mathrm{t}^{\mathrm{n}}}{\partial w^{n}}, D_{n t}=\frac{\partial \mathrm{t}^{\mathrm{n}}}{\partial w^{t}}, D_{t n}=\frac{\partial \mathrm{t}^{\mathrm{t}}}{\partial w^{n}}, D_{t t}=\frac{\partial \mathrm{t}^{\mathrm{t}}}{\partial w^{t}}
$$

The expressions of $D_{n n}, D_{n t}, D_{t n}$ and $D_{t t}$ are provided in Appendix 10. For the model M2, we obtain:

$$
\frac{\partial \mathbf{t}(\mathbf{w}, \boldsymbol{\alpha})}{\partial \mathbf{w}}=\left\{\begin{array}{l}
(51) \text { if } \mathrm{w}(\mathbf{x}, \mathrm{t}) \geq \alpha(\mathrm{t}) \\
\mathcal{K} \mathbf{1} \text { if } \mathrm{w}(\mathbf{x}, \mathrm{t})<\alpha(\mathrm{t})
\end{array}\right.
$$

where $\mathcal{K}$ has been defined in (44).

\section{A simple method for constructing the level-set function for ar- bitrary shaped inclusions in regular meshes}

A major difficulty when dealing with complex morphologies provided by voxelbased models (i.e. where each voxel is associated with a square element in $2 \mathrm{D}$ and to a cubic element in 3D) is to construct the level-set function $\phi(\mathbf{x})$. For example, Hamilton-Jacobi methods and upwind schemes [29] are very complex to implement and can fail for non-convex inclusions. To overcome these limitations, we propose a simple technique for constructing the levelset function for arbitrary shaped inclusions in regular meshes as provided in voxel-based models ${ }^{2}$. Let $\Omega^{i}$ a domain associated with inclusions such that $\Omega^{i} \subset \Omega$. We first solve the following problem:

\footnotetext{
$\overline{2}$ Note that we use abusively the term voxel, even though in $2 \mathrm{D}$ the term pixel would be more appropriate.
} 
Voxel of phase $1\left(\Omega^{\mathrm{i}}\right)$

- Voxel of phase $2(\Omega)$ Nodal interface $\tilde{\Gamma}$

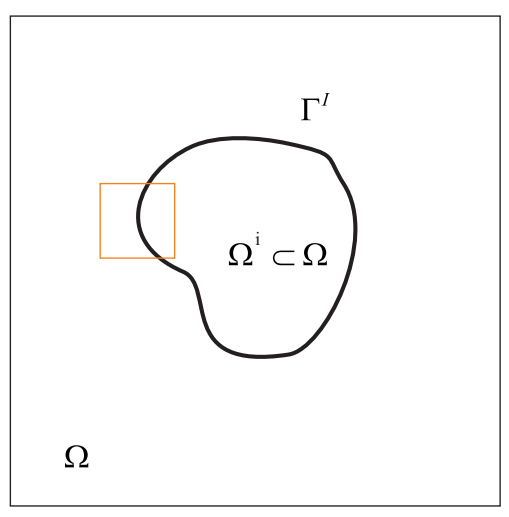

(a)

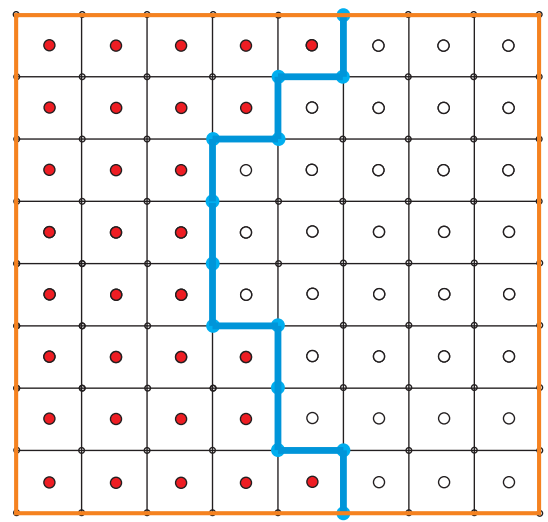

(b)

Fig. 4. (a) Schematic of the geometrical interfaces separating an inclusion $\Omega^{i}$ and the matrix; (b) interface nodes.

$$
\left\{\begin{array}{l}
\Delta \phi^{0}(\mathbf{x})+f(\mathbf{x})=0 \text { in } \Omega \\
\phi^{0}(x)=0 \text { in } \tilde{\Gamma} \\
\nabla \phi^{0}(\mathbf{x}) \cdot \mathbf{n}=0 \text { on } \partial \Omega
\end{array}\right.
$$

where $\tilde{\Gamma}$ is the interface composed by the set of nodes defined as follows (blue curve in Fig. 4 (b)). We detect the nodes which are at the corners of voxels on the interface by a simple algorithm (see Fig. 4): first, indices are assigned to each element, to indicate its belonging to the different phases. Second, for each node, we loop over the elements connected to the node. If at least two indices are different, then the node is defined as an interface node. In Fig. 5 we depict an inclusion defined in a voxel grid. In Fig. 5 (a), the nodes belonging to the interface are depicted in black. The source function $f(\mathbf{x})$ is defined as

$$
f(\mathbf{x})=\chi(\mathbf{x}) f_{1}+(1-\chi(\mathbf{x})) f_{2}
$$

$\chi(\mathbf{x})$ is a characteristic function such that $\chi(\mathbf{x})=1$ in $\Omega^{i}$ and zero elsewhere, and $f_{1}$ and $f_{2}$ are scalar parameters. The problem (56) is solved by finite elements. In the present test, we have used the following parameters $f_{1}=5$ and $f_{2}=-5$. In Fig. $5(\mathrm{~b})$, we show the obtained zero level of $\phi^{0}(\mathbf{x})$. We note that the obtained interface is not smooth, which can induce high local values of the gradient of $\phi(\mathbf{x})$ and numerical errors when evaluating the normal $\mathbf{n}^{I}$ and the displacement jump $\llbracket \mathbf{u}(\mathbf{x}) \rrbracket \simeq \mathbf{w}(\mathbf{x})$. We then propose the following correction step: for the nodes of the interface, we solve a second problem defined by: 


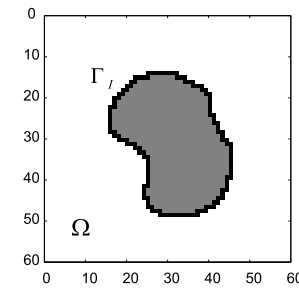

(a)

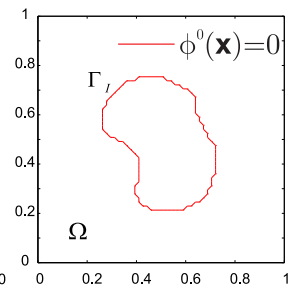

(b)

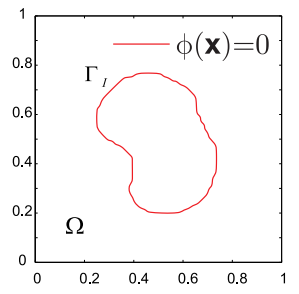

(c)

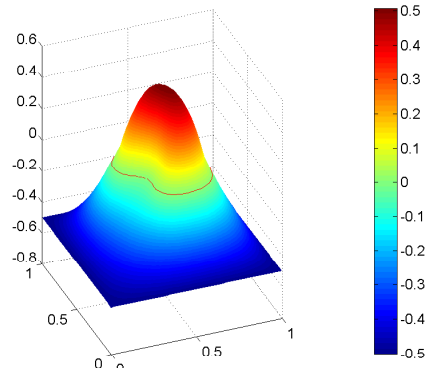

(d)

Fig. 5. Construction of the level-set function for an arbitrary geometry of inclusion: (a) nodes of the interface; (b) zero-level set obtained from the first step of the proposed algorithm; (c) zero-level set obtained from the second step of the proposed algorithm; (d) corresponding level-set function.

$$
\left\{\begin{array}{l}
\Delta \phi(\mathbf{x})+f(\mathbf{x})=0 \text { in } \Omega \\
\phi(x)=\phi^{0}(x) \text { in } \Omega / \tilde{\Gamma},
\end{array}\right.
$$

In practice, all nodes but the interface nodes are set with the values obtained in the problem (56). The only unknowns are the nodal values of the interface nodes. Then this step only requires to solve a small linear problem. In Fig. 5 (c), we show the zero level of the obtained level-set function $\phi(\mathbf{x})$, which presents a nice smooth boundary. Note that the choice of the ratio $f_{1} / f_{2}$ relies on the microstructural morphology, and more specifically on the number of voxels between two inclusions.

\section{Discretization and numerical implementation}

\subsection{FEM discretization of displacement problem}

A 2D plane strain FEM discretization is described in the following, even though extension to $3 \mathrm{D}$ is straightforward. The vector form of second-order tensors are introduced as $[\boldsymbol{\varepsilon}]=\left\{\varepsilon_{11} ; \varepsilon_{22} ; \sqrt{2} \varepsilon_{12}\right\}^{\mathrm{T}},[\boldsymbol{\sigma}]=\left\{\sigma_{11} ; \sigma_{22} ; \sqrt{2} \sigma_{12}\right\}^{\mathrm{T}}$, as well as the FEM approximations $\mathbf{u}=\mathbf{N} \mathbf{u}^{e}, \delta \mathbf{u}=\mathbf{N} \delta \mathbf{u}^{e}$, and $\Delta \mathbf{u}=\mathbf{N} \Delta \mathbf{u}^{e}$ where $\mathbf{u}^{e}, \delta \mathbf{u}^{e}$ and $\Delta \mathbf{u}^{e}$ are nodal displacement components in one element, nodal trial function components and nodal incremental displacement components, respectively. Furthermore, we have:

$$
[\varepsilon](\Delta \mathbf{u})=\mathbf{B}_{u} \Delta \mathbf{u}^{e}, \quad[\varepsilon](\delta \mathbf{u})=\mathbf{B}_{u} \delta \mathbf{u}^{e}
$$

where $\mathbf{B}_{u}$ is a matrix of shape function derivatives. From (12) the diffuse jump approximation vector $\mathbf{w}$ and its incremental counterparts can be discretized 
as:

$$
\mathbf{w}=h \mathbf{N} \tilde{\mathbf{B}}_{u} \mathbf{u}^{e}, \quad \Delta \mathbf{w}=h \mathbf{N B} \tilde{\mathbf{B}}_{u} \Delta \mathbf{u}^{e}, \quad \delta \mathbf{w}=h \mathbf{N} \tilde{\mathbf{B}}_{u} \delta \mathbf{u}^{e},
$$

where

$$
\mathbf{N}=\left[\begin{array}{cccc}
n_{1} & n_{2} & 0 & 0 \\
0 & 0 & n_{1} & n_{2}
\end{array}\right]
$$

and $n_{1}$ and $n_{2}$ are the $x$ - and $y$ - components of the normal vector $\mathbf{n}^{I}$ computed from (9), and based on the level-set function $\phi$ constructed as described in the previous section, and $\tilde{\mathbf{B}}_{u}$ is a matrix of shape functions derivatives such that

$$
\left[\begin{array}{c}
\frac{\partial u_{1}}{\partial x_{1}} \\
\frac{\partial u_{1}}{\partial x_{2}} \\
\frac{\partial u_{2}}{\partial x_{1}} \\
\frac{\partial u_{2}}{\partial x_{2}}
\end{array}\right]=\tilde{\mathbf{B}}_{u} \mathbf{u}^{e} .
$$

We define the vector associated with $\tilde{\varepsilon}$ by:

$$
[\tilde{\boldsymbol{\varepsilon}}]=\left[\begin{array}{c}
\tilde{\varepsilon}_{11} \\
\tilde{\varepsilon}_{22} \\
\sqrt{2} \tilde{\varepsilon}_{12}
\end{array}\right]=\gamma_{\beta}(\mathbf{x})\left[\begin{array}{c}
w_{1} n_{1} \\
w_{2} n_{2} \\
\frac{1}{\sqrt{2}}\left(w_{1} n_{2}+w_{2} n_{1}\right)
\end{array}\right]
$$

Then we have:

$$
[\tilde{\varepsilon}(\Delta \mathbf{u})]=h \gamma_{\beta}(\mathbf{x}) \mathbf{M} \tilde{\mathbf{B}}_{u} \Delta \mathbf{u}^{e}
$$

where

$$
\mathbf{M}=\left[\begin{array}{cccc}
n_{1}^{2} & n_{1} n_{2} & 0 & 0 \\
0 & 0 & n_{1} n_{2} & n_{2}^{2} \\
\frac{1}{\sqrt{2}} n_{1} n_{2} & \frac{1}{\sqrt{2}} n_{2}^{2} & \frac{1}{\sqrt{2}} n_{1}^{2} & \frac{1}{\sqrt{2}} n_{1} n_{2}
\end{array}\right] .
$$

After discretization, the linear system (49) reduces to the set of linear algebraic equations:

$$
\mathbf{K}_{\text {tan }} \Delta \tilde{\mathbf{u}}=-\mathbf{R}\left(\tilde{\mathbf{u}}^{k}\right)
$$


where $\tilde{\mathbf{u}}$ is a column vector containing the nodal values of $\mathbf{u}$ and

$$
\begin{aligned}
& \mathbf{K}_{t a n}=\int_{\Omega}\left[\mathbf{B}_{u}^{T}-h \gamma_{\beta}(\mathbf{x}) \tilde{\mathbf{B}}_{u}^{T} \mathbf{M}^{T}\right] \mathbf{C}(\mathbf{x})\left[\mathbf{B}_{u}-h \gamma_{\beta}(\mathbf{x}) \mathbf{M} \tilde{\mathbf{B}}_{u}\right] d \Omega \\
& +\int_{\Omega} h^{2} \gamma_{\beta}(\mathbf{x}) \tilde{\mathbf{B}}_{u}^{T} \mathbf{N}^{T} \mathbf{K}_{I} \mathbf{N} \tilde{\mathbf{B}}_{u} d \Omega,
\end{aligned}
$$

and

$$
\begin{aligned}
& \mathbf{R}=\int_{\Omega}\left[\mathbf{B}_{u}^{T}-h \gamma_{\beta}(\mathbf{x}) \tilde{\mathbf{B}}_{u}^{T} \mathbf{M}^{T}\right] \mathbf{C}(\mathbf{x})\left[\mathbf{B}_{u}-h \gamma_{\beta}(\mathbf{x}) \mathbf{M} \tilde{\mathbf{B}}_{u}\right]\left(\mathbf{u}^{e}\right)^{k} d \Omega \\
& +\int_{\Omega} h \gamma_{\beta}(\mathbf{x}) \tilde{\mathbf{B}}_{u}^{T} \mathbf{N}^{T} \mathbf{t}\left(\mathbf{w}^{k}\right) d \Omega+\int_{\Omega} \mathbf{f} \mathbf{N}^{T} d \Omega+\int_{\Omega_{F}} \overline{\mathbf{F}} \mathbf{N}^{T} d \Gamma
\end{aligned}
$$

In Eq. (67), $\mathbf{C}$ is the matrix form corresponding to the fourth-order tensor $\mathbb{C}$ in Eq. (52).

\subsection{FEM discretization of the phase field problem}

As we employ a staggered procedure, we solve alternatively the phase field problem and then the mechanical problem. Given displacements from the mechanical problem, the phase field problem is linear. The phase field and phase field gradient are approximated in one element by

$$
d(\mathbf{x})=\mathbf{N}_{d}(\mathbf{x}) \mathbf{d}^{e} \quad \text { and } \quad \nabla d(\mathbf{x})=\mathbf{B}_{d}(\mathbf{x}) \mathbf{d}^{e},
$$

where $\mathbf{N}_{d}(\mathbf{x})$ and $\mathbf{B}_{d}(\mathbf{x})$ are vectors and matrices of shape functions and of shape functions derivatives, respectively, and $\mathbf{d}^{e}$ are the nodal values of $\mathbf{d}_{n+1}$.

The same discretization is employed for the test function:

$$
\delta d(\mathbf{x})=\mathbf{N}_{d}(\mathbf{x}) \delta \mathbf{d}^{e} \quad \text { and } \quad \nabla \delta \mathrm{d}(\mathbf{x})=\mathbf{B}_{\mathrm{d}}(\mathbf{x}) \delta \mathbf{d}^{\mathrm{e}} .
$$

Introducing the above FEM discretization in (36) results into a linear system of equations:

$$
\mathbf{K}_{d} \tilde{\mathbf{d}}=\mathbf{F}_{d}
$$

where $\tilde{\mathbf{d}}$ is a column vector containing the nodal values of $\mathbf{d}$ and

$$
\mathbf{K}_{d}=\int_{\Omega}\left\{\left(\frac{g_{c}}{l}(1-\beta)+2 \mathcal{H}\right) \mathbf{N}_{\mathbf{d}}^{\mathbf{T}} \mathbf{N}_{\mathbf{d}}+(1-\beta) g_{c} l \mathbf{B}_{d}^{T} \mathbf{B}_{d}\right\} d \Omega
$$

and

$$
\mathbf{F}_{d}=\int_{\Omega} 2 \mathbf{N}_{d}^{T} \mathcal{H}\left(\mathbf{u}_{n}\right) d \Omega
$$




\subsection{Overall algorithm}

The overall algorithm is described in the following.

\section{- Initialization}

1.1 Initialize the displacement field $\mathbf{u}_{0}(\mathbf{x})$, the phase field $d_{0}(\mathbf{x})$, and the strain-history functional $\mathcal{H}_{0}=0$.

1.2 Compute the level-set function $\phi(\mathbf{x})$ by means of the algorithm described in section 4 .

1.3 Compute the phase field $\beta(\mathbf{x})$ by solving (5).

- FOR all loading increment (pseudo time $t^{n+1}$ ):

Given $\mathbf{u}_{n}, d_{n}$ and $\mathcal{H}_{n}(\mathbf{x})$ :

2.1 Compute the strain history functional $\mathcal{H}_{n+1}(\mathbf{x})$ by $(34)$.

2.2 Compute $d_{n+1}(\mathbf{x})$ by solving the linear phase field problem (71).

2.3 Compute $\mathbf{u}_{n+1}(\mathbf{x})$ :

Initialize $\mathbf{u}_{k}=\mathbf{u}_{n}$ (displacement of time $t_{n}$ )

WHILE $\left\|\Delta \mathbf{u}_{k+1}\right\|>\epsilon, \epsilon<<1$ :

2.3.1 Compute $\Delta \mathbf{u}_{k+1}^{e}$ from (66).

2.3.2 Update $\mathbf{u}_{k+1}=\mathbf{u}_{k}+\Delta \mathbf{u}_{k+1}^{e}$.

2.3.3 $(.)_{n} \leftarrow(.)_{n+1}$ and go to 2.3.1.

END

\section{END}

\section{$6 \quad$ Numerical examples}

For all of the following numerical examples, a regular mesh of bilinear 4-node elements and plane strain assumption has been used. We assume a simplified cohesive model for the interface by taking into account the normal traction only, $\mathbf{t}(w) \cdot \mathbf{n}^{I}=t^{n}$. Then, the cohesive law can be rewritten as:

$$
t^{n}=g_{c}^{I}\left(\frac{\mathrm{w}^{\mathrm{n}}}{\delta^{n}}\right) \exp \left(-\frac{\mathrm{w}^{\mathrm{n}}}{\delta^{\mathrm{n}}}\right) .
$$

\subsection{Discontinuous benchmark}

In this first example, we analyze the accuracy of the diffuse displacement jump approximation introduced in section 2.2. For this purpose, a benchmark with analytical solution is studied. A bi-material square domain including a cohesive zone is depicted in Fig. 6. The length of the square domain is $L=1$ $\mathrm{mm}$. 


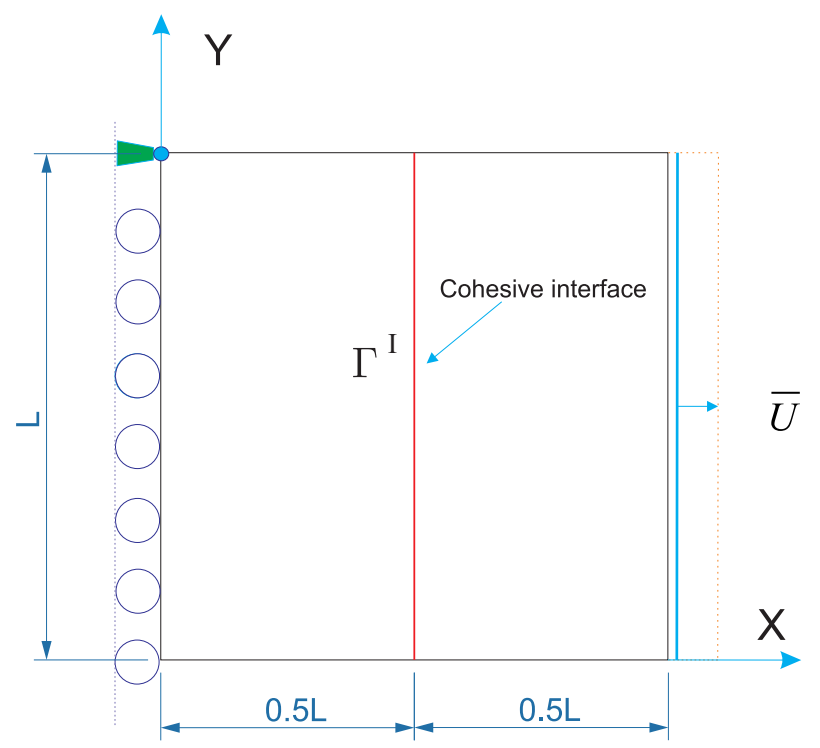

Fig. 6. Discontinuous benchmark, geometry and boundary conditions.

We consider small strains, and zero Poisson's coefficient $\nu=0$ in both parts of the domain. For both (left and right) parts, isotropic elastic behavior is assumed, with Young's coefficients $E_{1}=100 \mathrm{Mpa}$ and $E_{2}=200 \mathrm{Mpa}$ for the respective domains. With the boundary conditions described in Fig. 6 the problem is unidimensional with a solution independent on the $y$-component (the displacements are prescribed along the $x$-direction only, the displacement along $y$ are free). We use here a linear cohesive law to consider the first step of interface opening:

$$
t_{n}=\frac{g_{c}^{I}}{\left(\delta^{n}\right)^{2}} \times \llbracket u_{1} \rrbracket
$$

The analytical solution for this problem can be simply obtained by considering the displacement boundary conditions and the continuity condition at the interface, and is provided in Appendix 9.

First, we evaluate the error of the displacement jump approximation introduced in Eq. (12). A $500 \times 500$ mesh was chosen, and the jump approximation parameter $h$ in Eq. (12) varies between $0.25 \leq h_{e} / h \leq 10$. We define the relative jump error with respect to the analytical solution as:

$$
E R R_{1}=\sqrt{\frac{\int_{\Gamma}\left(\llbracket \mathbf{u}^{h}(\mathbf{x}) \rrbracket-\llbracket \mathbf{u}^{e x}(\mathbf{x}) \rrbracket\right) \cdot\left(\llbracket \mathbf{u}^{h}(\mathbf{x}) \rrbracket-\llbracket \mathbf{u}^{e x}(\mathbf{x}) \rrbracket\right) d \Gamma}{\int_{\Gamma}\left(\llbracket \mathbf{u}^{e x}(\mathbf{x}) \rrbracket\right) d \Gamma}} .
$$

with $\left[\left[\mathbf{u}^{h}(\mathbf{x})\right]\right]=\mathbf{w}(\mathbf{x})$ is given by Eq. (12). We consider here undamaged bulk materials $(d(\mathbf{x})=0)$ everywhere. Numerical parameters of the constitutive relation of the interface are: $t_{u}=1 \mathrm{MPa}$ and $g_{c}^{I}=0.1 \mathrm{~N} / \mathrm{mm}$. Results are depicted in Fig. 7. We note minimal error for the ratio $h_{e} / h$. 


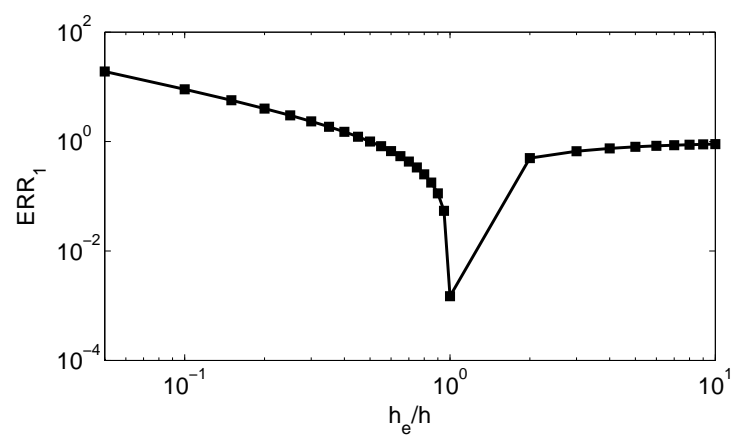

Fig. 7. Displacement jump error: influence of the parameter $h$ in Eq. (12) with respect to the mesh size $h_{e}$.

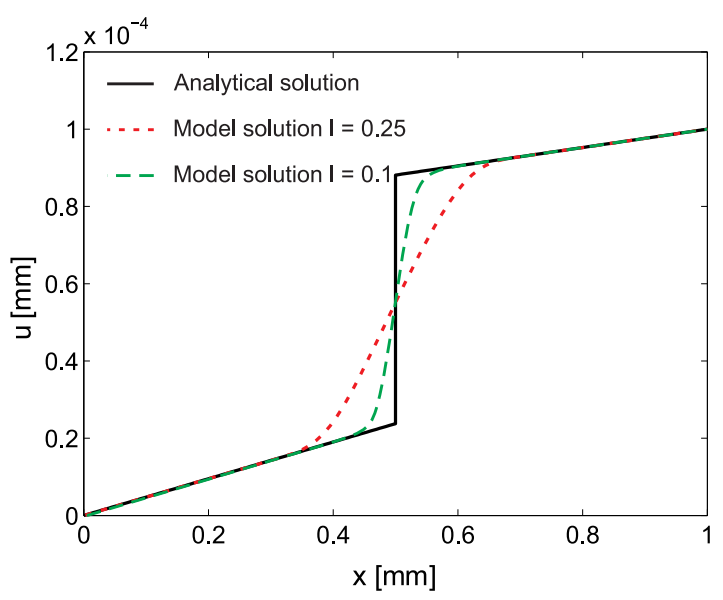

Fig. 8. Discontinuous benchmark problem: comparison between the analytical solution and the approximated one for two values of the regularization parameter $l$.

Next, we analyze more specifically the influence of the regularization parameter $l$. A $1000 \times 1000$ mesh with an element size $h_{e}$ was chosen to ensure that $l / h_{e} \geq 1$, and the approximation jump in (12) parameter is chosen $h=h_{e}$ for all tested values of $l_{\beta}$. The regularization parameter $l$ takes values between 0.002 and 0.02. We remind that in this test, the phase-field is set to zero $d(\mathbf{x})=0$ and the discontinuity only occurs from the cohesive laws at the interface. Comparisons of the displacement solution for several values of $l$ is provided in Fig. 8.

We define the relative regularization error by:

$$
E R R_{2}=\frac{\left\|\mathbf{u}^{h}(\mathbf{x})-\mathbf{u}^{e x}(\mathbf{x})\right\|_{L^{2}}}{\left\|\mathbf{u}^{e x}(\mathbf{x})\right\|_{L^{2}}}=\sqrt{\frac{\int_{\Omega}\left(\mathbf{u}^{h}(\mathbf{x})-\mathbf{u}_{\mathrm{ex}}(\mathbf{x})\right) \cdot\left(\mathbf{u}^{h}(\mathbf{x})-\mathbf{u}_{\mathrm{ex}}(\mathbf{x})\right) d \Omega}{\int_{\Omega}\left(\mathbf{u}_{\mathrm{ex}}(\mathbf{x})\right) \cdot\left(\mathbf{u}_{\mathrm{ex}}(\mathbf{x})\right) d \Omega}}
$$

The global $L_{2}$ error norm of the regularized solutio for the benchmark problem is plotted in Fig. 9. 


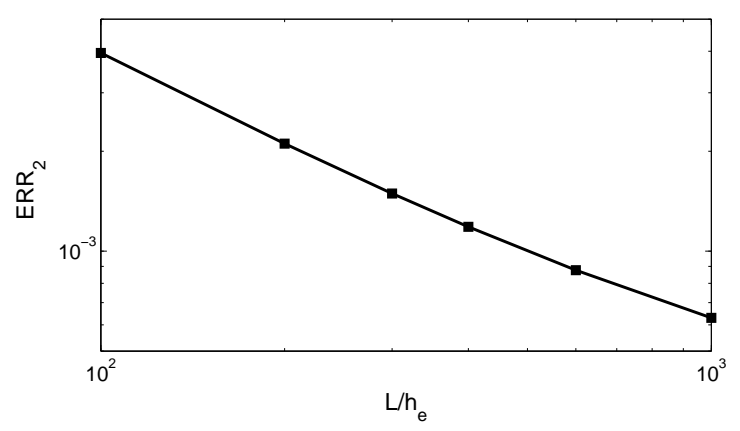

Fig. 9. $L_{2}$ error norm for the discontinuous benchmark problem.

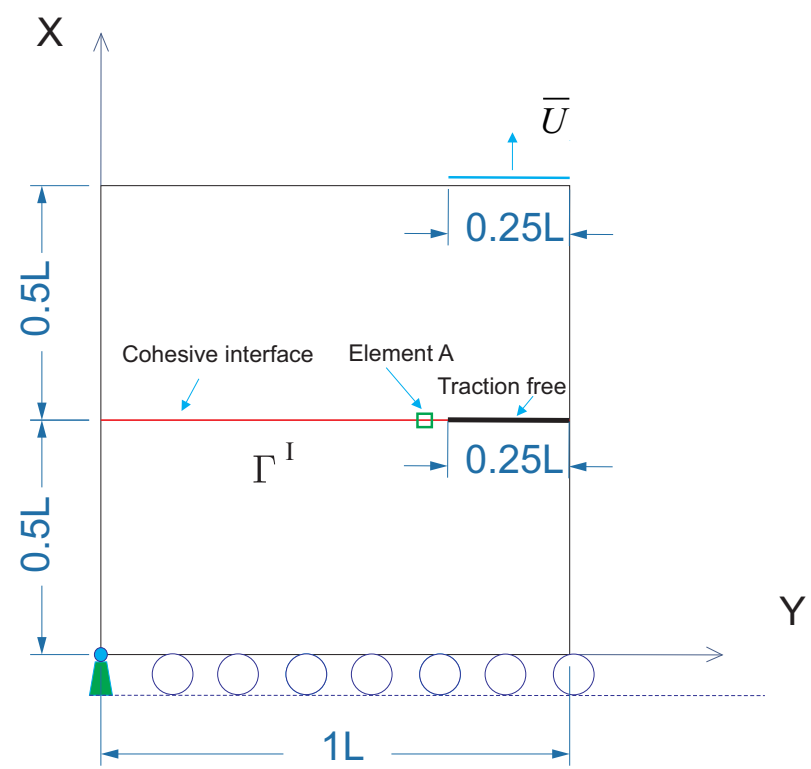

Fig. 10. Geometry of the sample for the fatigue crack test: geometry and boundary conditions.

\subsection{Fatigue cracking}

In this next example, we investigate the capability of the present method for handling fatigue cracking under cyclic loading. A square domain of length $L=$ $1 \mathrm{~mm}$ is considered. The domain contains a cohesive interface, whose geometry is depicted in Fig. 10.

The material is described by the model described in section 3.1 with parameters $E=100 \mathrm{MPa}$ and $\nu=0$, fracture strength and toughness $t_{u}=10 \mathrm{MPa}$ and $g_{c}=g_{c}^{I}=0.1 \mathrm{~N} / \mathrm{mm}$, respectively. Note that from now on, we will assume that $t^{n}=0$. In addition, small strain an local isotropic behavior of the phases is assumed. The regularization parameter $l$ is chosen as $l=0.02 \mathrm{~mm}$. Planestrain condition is assumed. The two models M1 and M2 have been used for comparison. A cyclic displacement $\bar{U}$ whose evolution is described in Fig. 11 (a) is prescribed on a portion of the upper end, as depicted in Fig. 10. The 


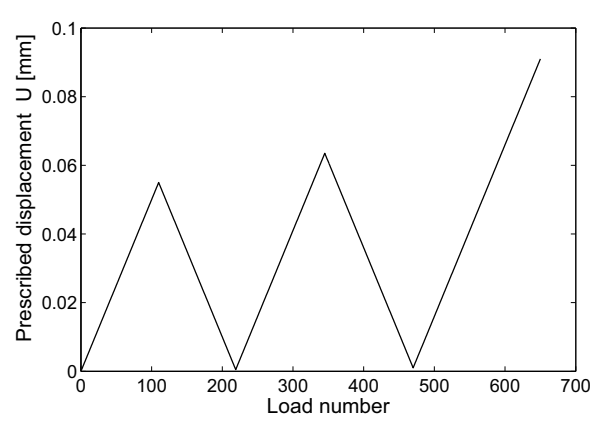

(a)

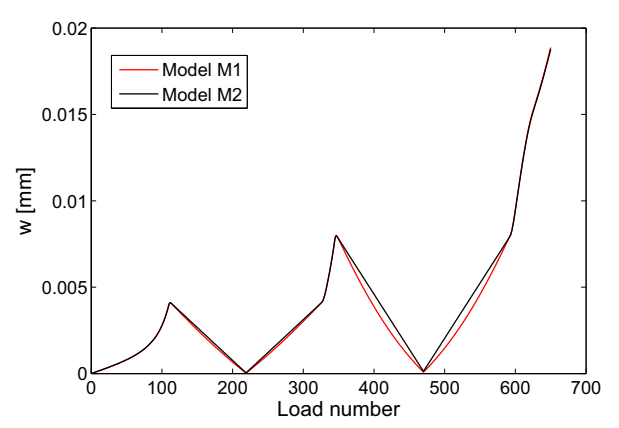

(b)

Fig. 11. Fatigue crack test: (a) evolution of the load; (b) Computed displacement jump along $y$.

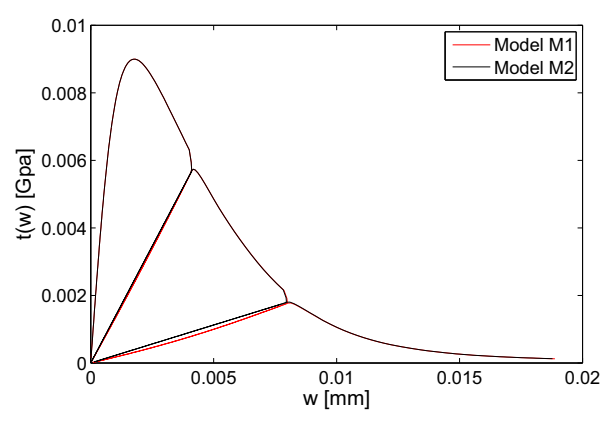

(a)

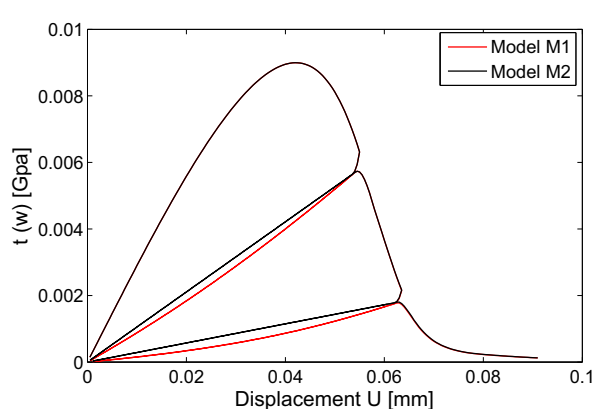

(b)

Fig. 12. Fatigue crack test: (a) cohesive traction with respect to the displacement jump within the interface; (b) cohesive traction within the interface with respect to the prescribed displacement.

displacements are prescribed along the $y$-direction while the displacement along $x$ are free. The evolution of the computed displacement jump along $y$ in the element A near the crack tip is depicted in Fig. 11 (b) for both models M1 and M2.

The normal traction force in the element located near the crack tip is depicted in Fig. 12 (a) versus displacement jump and (b) versus the prescribed vertical displacement. We can note that simplest model M1 is able to capture the interfacial damage, even without using internal variables. The response is very similar to the response obtained by model M2.

\subsection{Crack propagation in symmetric three-point bending test}

The purpose of this example is to validate the solution provided by our new formulation. We consider the three-point bending problem studied e.g. in [41] and described Fig. 13. The dimensions of the beam are $L=10 \mathrm{~mm}$, and $H=3 \mathrm{~mm}$. The load consists into a prescribed displacement at the center of 


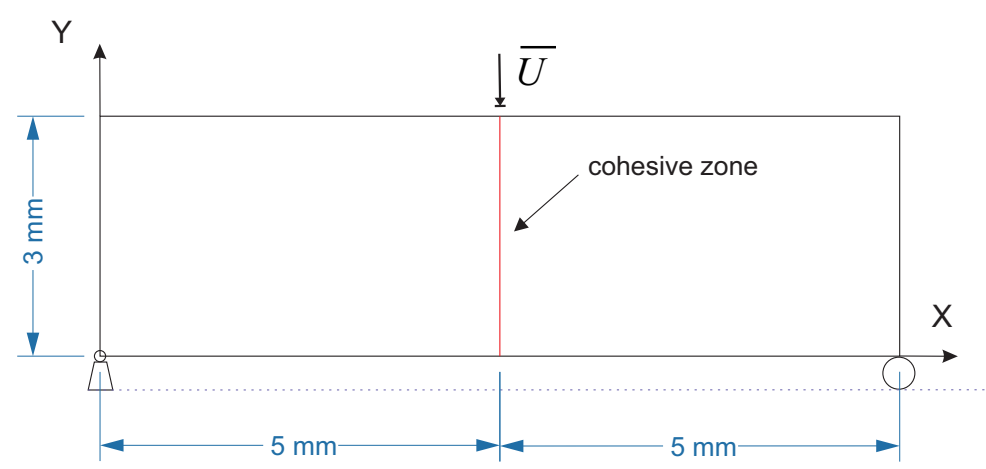

Fig. 13. Symmetric three-point bending test problem: Geometry and boundary conditions.

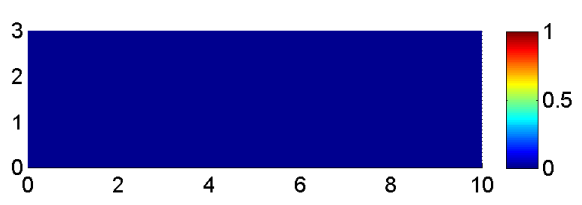

(a)

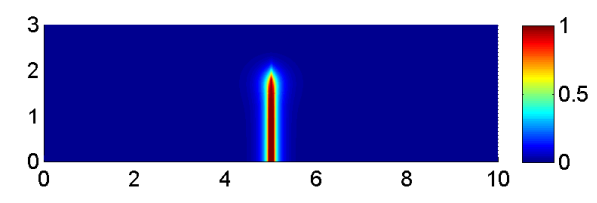

(c)

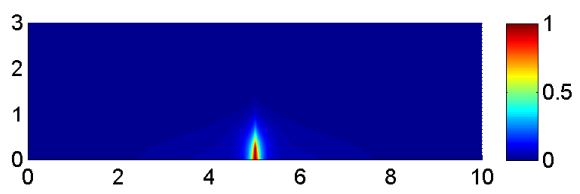

(b)

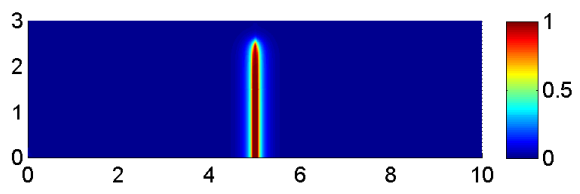

(d)

Fig. 14. Symmetric three-point bending test problem: Damage evolution for: $\bar{U}=0$ $\mathrm{mm} ; \bar{U}=0.2 \mathrm{~mm} ; \bar{U}=0.25 \mathrm{~mm}$ and $\bar{U}=0.35 \mathrm{~mm}$

the beam on the top edge. The node at $(x=0),(y=0)$ is fixed, while the node at $(x=L),(y=0)$ the $y$-displacement is fixed and the $x$-displacement is free. For this case, an initial cohesive interface has been inserted as described in Fig. 13, to validate the cohesive model only. Materials on either sides of the interface are identical.

A regular mesh of $60 \times 200$ quadrilateral elements is employed. The material parameters are chosen as follows: $E=100 \mathrm{MPa}, \nu=0.0$, the fracture strength is $t_{u}=1.0 \mathrm{Mpa}$, and the toughness is $g_{c}=g_{c}^{I}=0.1 \mathrm{~N} / \mathrm{mm}$. The computation is performed with monotonic displacement increments of $\bar{U}=5.10^{-3} \mathrm{~mm}$ for 120 load increments. The displacements are prescribed along the $y$-direction while the displacement along $x$ are free. The regularization parameter $l$ is chosen as $l=0.15 \mathrm{~mm}$. Plane strain condition is assumed. The damage evolution (phase field $d(\mathbf{x})$ ) for the different values of the load is depicted in figure 14 . In contrast to the approach developed in [12] where interfaces are initially damaged in the phase field sense, here the phase field can be used to follow the interfacial damage directly. 


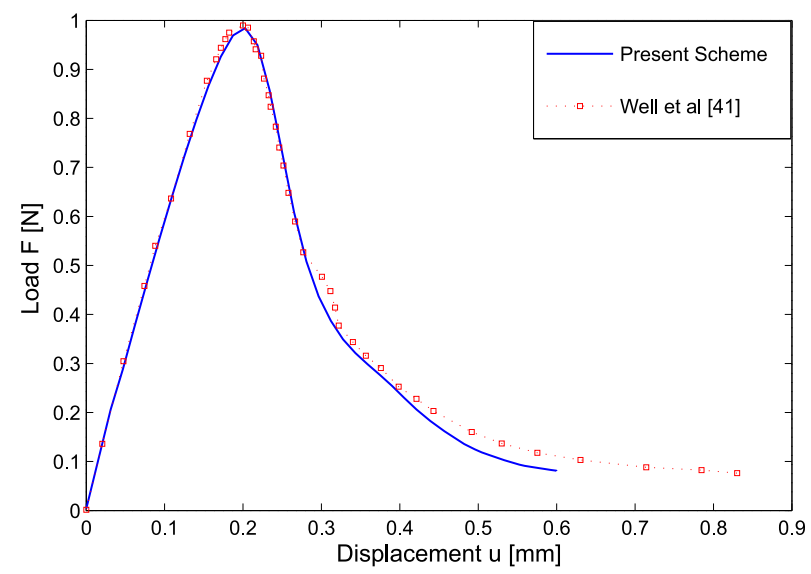

Fig. 15. Force-displacement curve for the crack propagation problem with symmetric three-point bending test problem: comparison between the present approach and the results obtained in [3]

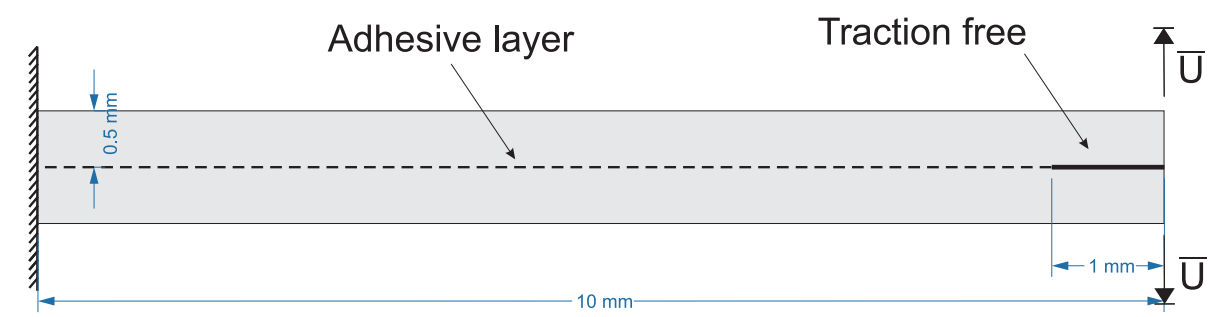

Fig. 16. Delamination peel test problem: geometry and boundary conditions

To validate the results, we compare in Fig. 15 the curve obtained with the present method to the solution provided in [41]. We can note a good agreement between both simulations which validates the present method for the damage of interfaces.

\subsection{Delamination peel test}

In this next test, another validation is performed through the so-called peeling test, described in Fig. 16: two cantilever elastic beams are connected over $90 \%$ of their length by means of an adhesive layer. The structure is $10 \mathrm{~mm}$ in length and $0.5 \mathrm{~mm}$ in height (see Fig. 16).

The points located at the top and bottom right ends of the structure are subjected to a prescribed vertical displacement $\bar{U}$. The bulk material is modeled as linear elastic and isotropic with Young's modulus $E=1000 \mathrm{MPa}$ and Poisson's ratio $\nu=0.3$. The fracture strength and fracture energy are taken as $t_{u}=1 \mathrm{MPa}$ and $g_{c}=g_{c}^{I}=0.1 \mathrm{~N} / \mathrm{mm}$, respectively. The length scale parameter $l$ is chosen $l=0.05 \mathrm{~mm}$. Plane strain conditions are assumed. In Fig. 17, the evolution of the interfacial damage is depicted. In the present framework, this damage coincides with the phase field distribution, which is initially zero 

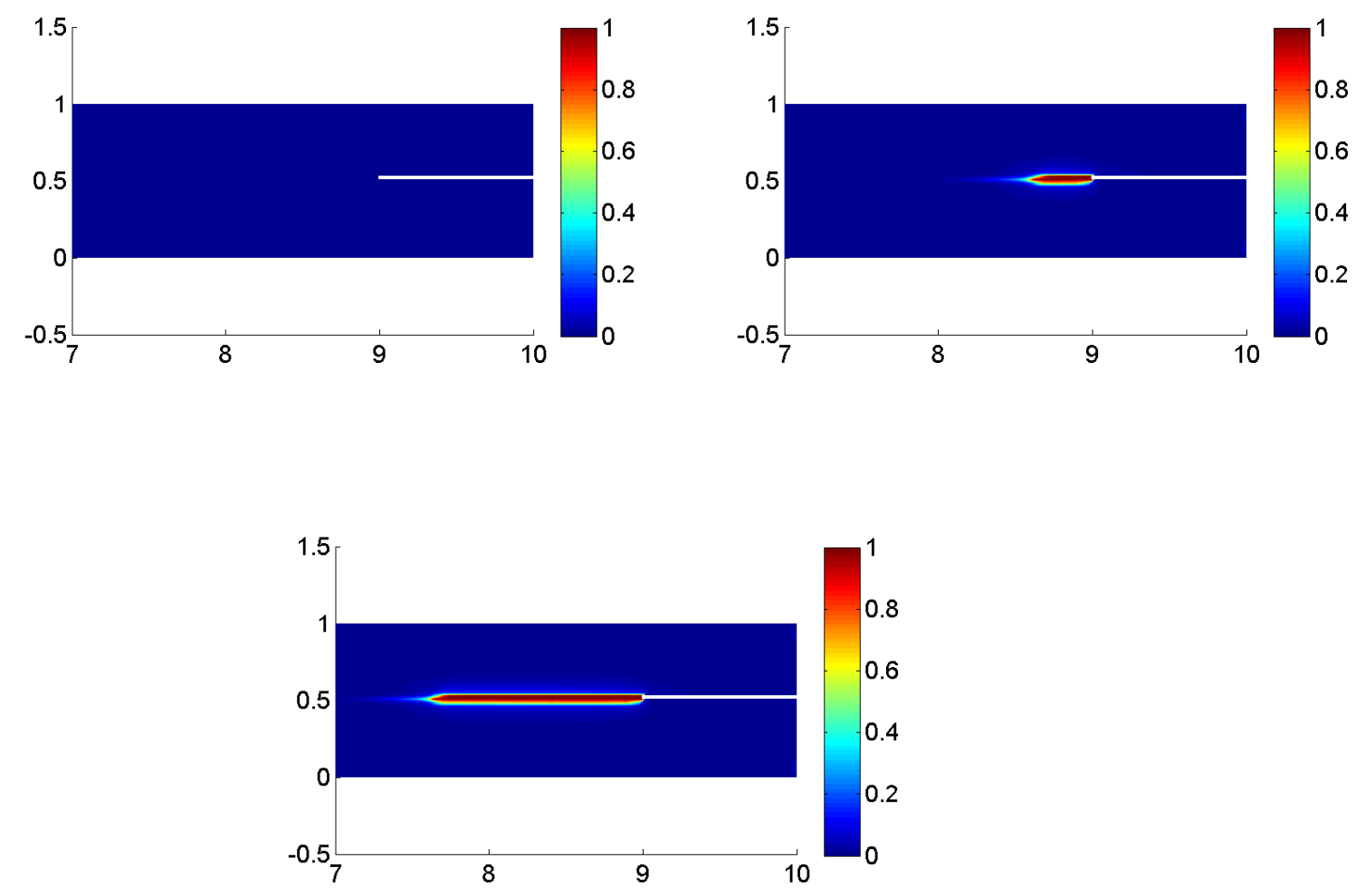

Fig. 17. Delamination peel test problem: phase field evolution $d(\mathbf{x})$ for: $u=0 \mathrm{~mm}$, $u=0.4 \mathrm{~mm}$ and $u=1 \mathrm{~mm}$

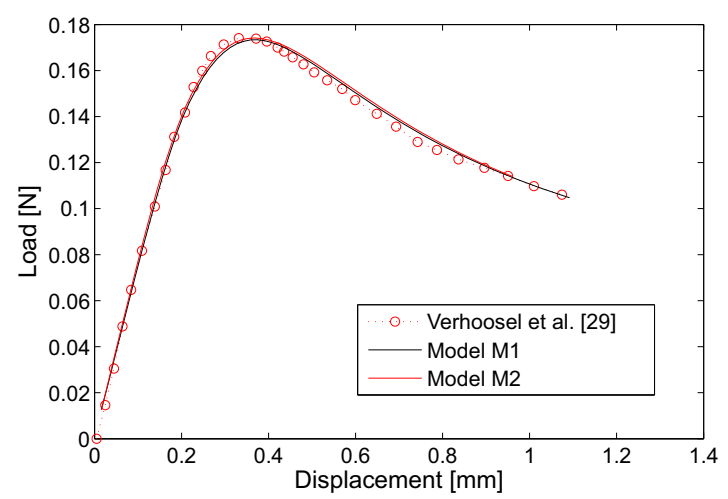

Fig. 18. Force-displacement curve for the peel-test problem: comparison between the present approach and the results obtained in [39].

in the interface, in contrast to the approach proposed in [39].

To validate the results, we compare in Fig. 18 the obtained response with the solution in [39] for both models M1 and M2. We can note that both models provide a very good agreement with the reference solution. 


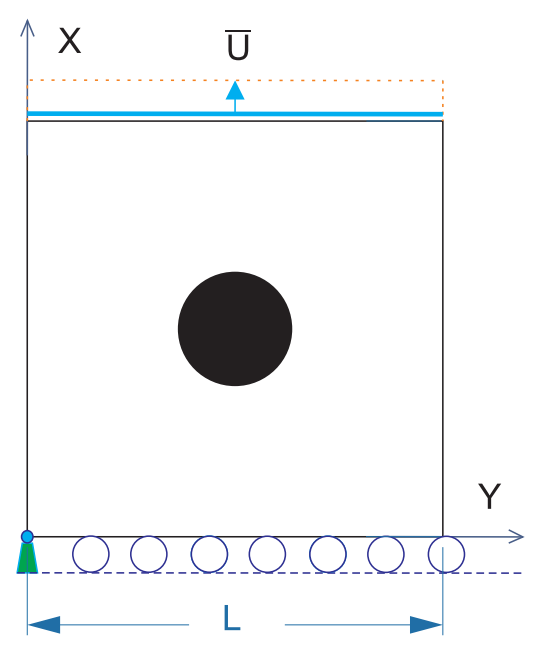

(a)

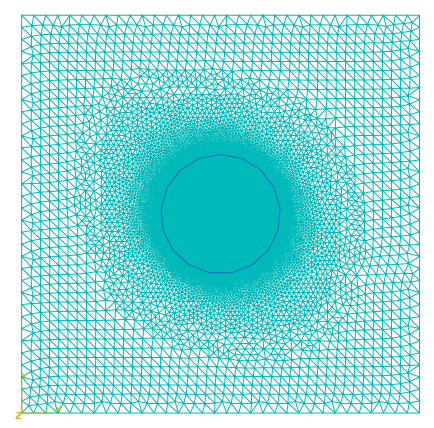

(b)

(a)

Fig. 19. Traction test of a microstructure containing a single inclusion with damageable interface: (a) geometry of the phases; (b) unstructured mesh of triangles.

\subsection{Traction test of a microstructure with circular inclusion}

In this example, we test the influence of the mesh on the predicted crack path, when an interfacial crack interact with a bulk crack. The problem geometry is depicted in Fig. 19. A square domain of length $L=1 \mathrm{~mm}$ contains a centered circular inclusion of diameter $D=0.3 L$.

The boundary conditions are as follows: on the lower end $(y=0)$, the $y-$ displacements are fixed, while the $x$-displacements are free and the node $(x=0, y=0)$ is fixed. On the upper end, the $x$-displacements are free, while the $y$-displacements are prescribed to an increasing uniform value of $\bar{U}$ during the simulation. Plane strain is assumed.

The material parameters of each phase are taken as: $E_{i}=52 \mathrm{GPa}, \nu_{i}=0.3$, $E_{m}=10.4 \mathrm{GPa}, \nu_{m}=0.3$, where the indices $i$ and $m$ correspond to the matrix and inclusion, respectively. These parameters are those of a mortar composed of a cement paste (matrix) and sand (inclusions) [17]. The toughness is $g_{c}=g_{c}^{I}=1 \times 10^{-4} \mathrm{kN} / \mathrm{mm}$ and $t_{u}=10^{-2} \mathrm{GPa}$. Computations are performed with monotonic displacement increments of $\bar{U}=5 \cdot 10^{-5} \mathrm{~mm}$ during 260 load increments for interfacial model, and 320 load increments for the classical phase field method.

To investigate the effects of the mesh on the crack path and load response, 4 different meshes have been used for comparison: 3 regular meshes wit 4-node elements on regular grids of $200 \times 200,300 \times 300$ and $400 \times 400$ element, and a mesh of 30488 elements with $h_{e}^{\max }=0.03 \mathrm{~mm}$ and $h_{e}^{\min }=0.003 \mathrm{~mm}$. The 


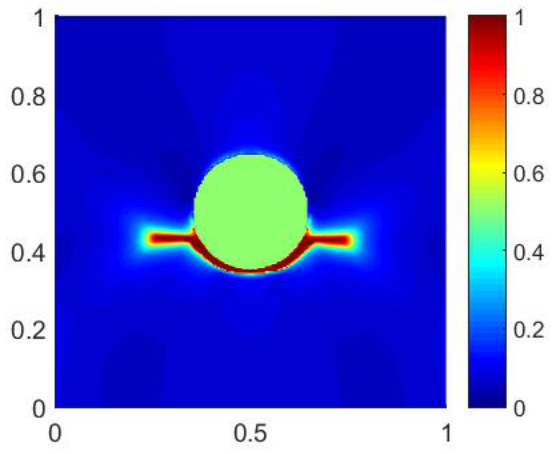

(a)

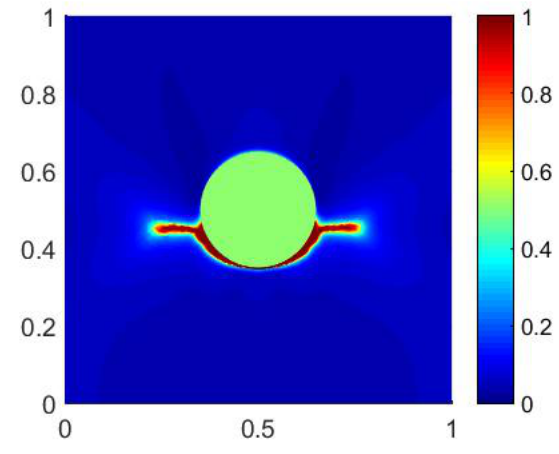

(b)

Fig. 20. (a) Crack path obtained for a regular mesh. (b) Crack path obtained for an unstructured mesh. The load is $\bar{U}=0.0107 \mathrm{~mm}$.

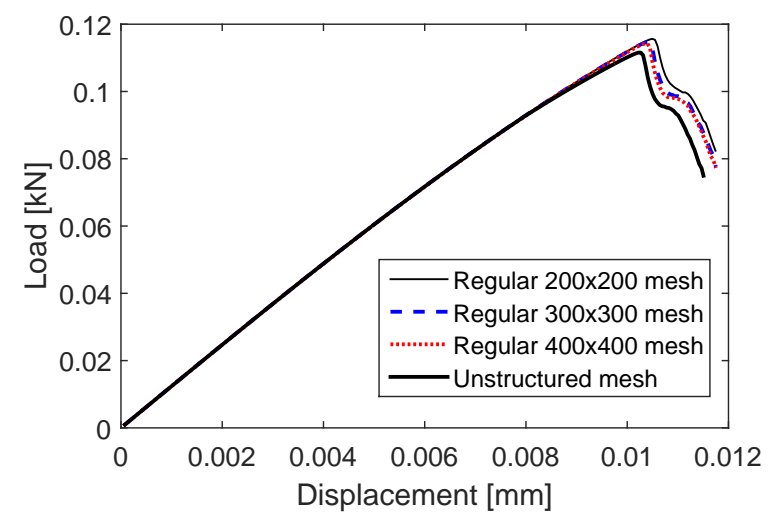

Fig. 21. Comparison of solutions obtained with different meshes for the single inclusion problem with damageable interface.

obtained crack paths are depicted in Fig. 20 for the regular mesh of $200 \times 200$ elements and the unstructured mesh, which have roughly the same number of elements. The comparison has been made for the same load: $\bar{U}=0.01175$ $\mathrm{mm}$. We can note a similar crack path for both meshes. Finally, the response for the 4 meshes are compared in Fig. 21, which shows the convergence of the solution with mesh refinement. Note that the unstructured mesh solution corresponds to the element size of the $200 \times 200$ regular mesh. Here, we have chosen $h=h_{e}$. We can see from Fig. 21 that as the mesh is being refined, the solution converges also with respect to the value of $h$.

Next, we investigate the influence of modifying the parameter $l_{\beta}$ in (5). We chose the following values: $l_{d} / l_{\beta}=1 / 3, l_{d} / l_{\beta}=1$ and $l_{d} / l_{\beta}=2$ for $l=0.013$ $\mathrm{mm}$. Results are presented in Figs. 22 and 23 for the same load $\bar{U}=0.0107$ $\mathrm{mm}$, showing that a modification of $l_{\beta}$ has a strong influence on the crack path and on the overall response. This suggests that like $l_{d}$, this parameter should be identified through experimental data. However, as the purpose of this work is to demonstrate the capabilities of the numerical scheme, we will assume in 


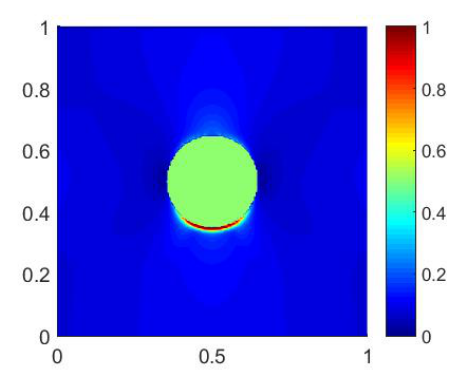

(a)

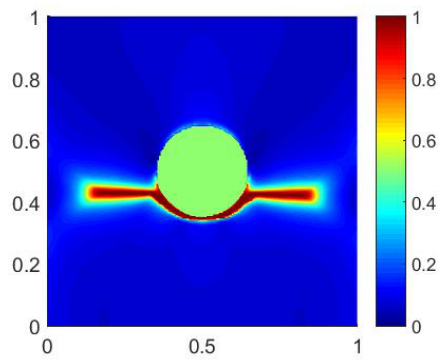

(b)

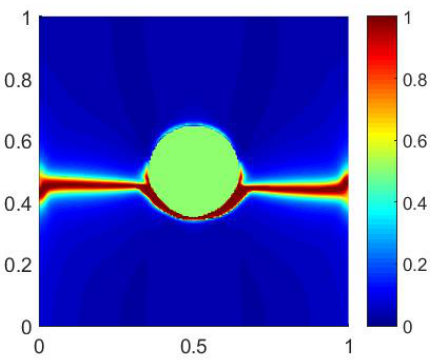

(c)

Fig. 22. Crack paths obtained with different length parameter $l_{\beta}$ for the same load $\bar{U}=0.0107 \mathrm{~mm}$ : (a) $l_{d} / l_{\beta}=1 / 3$; (b) $l_{d} / l_{\beta}=1 ; l_{d} / l_{\beta}=2, l=0.013 \mathrm{~mm}$.

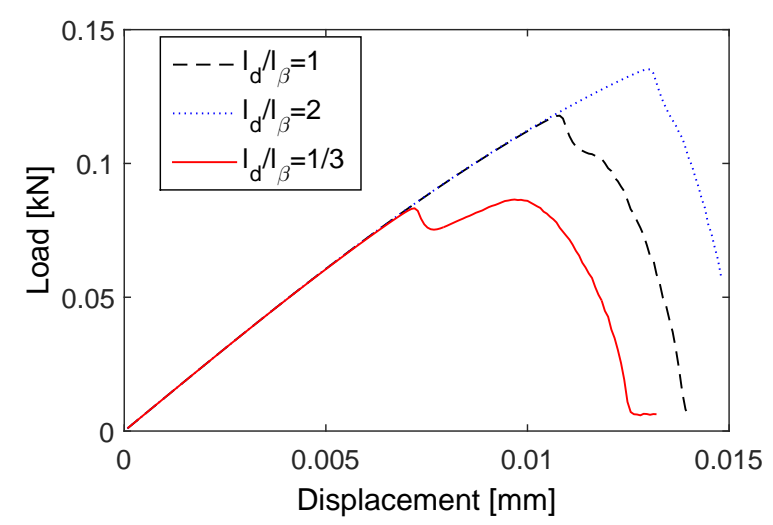

Fig. 23. Comparison of solutions obtained with different length parameters $l_{\beta}$.

the following that $l_{d}=l_{\beta}=l$.

\subsection{Traction test of a microstructure with non convex inclusion}

In this next test, we evaluate the capability of the method to describe interfacial damage in a microstructure containing an inclusion with complex, non-convex geometry. In addition, the initial data of local properties are provided on a regular grid of voxels. We then test the procedure described in section 4 to construct the level-set function used to evaluate the normal vector to the interface and construct the smeared discontinuous field $\llbracket \mathbf{u}(\mathbf{x}) \rrbracket$. The geometry of the microstructure is described in Fig. 24 (a). A non-convex inclusion composed of the union of two discs is embedded into a square domain of length $L=1 \mathrm{~mm}$. This non convex geometry has been chosen to test the capability of the level-set construction method described in section 4 for this difficult case. The obtained level-set is plotted in Fig. 24 (b) and its zero isocontour, corresponding to the interface geometry, is depicted in Fig. 24 (c). The geometrical parameters are: $A=0.43 L, D=0.2 L$. A small dissymmetry is introduced through the parameter $\epsilon=0.01 \mathrm{~L}$. 


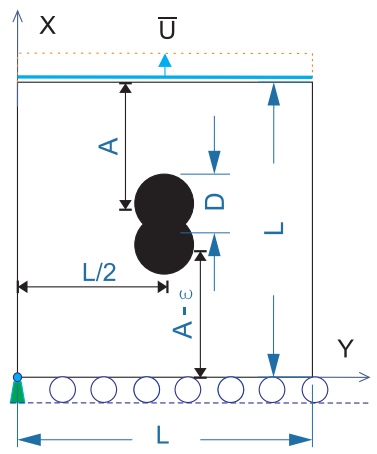

(a)

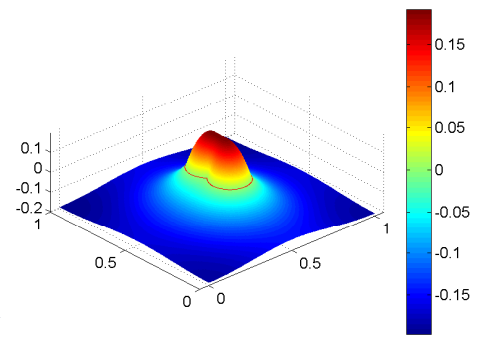

(b)

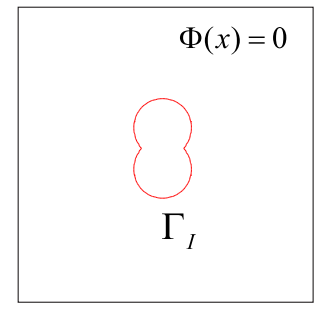

(c)

Fig. 24. Traction test of a complex microstructure in 2D: (a) geometry of the phases; (b) and (c) level-set function

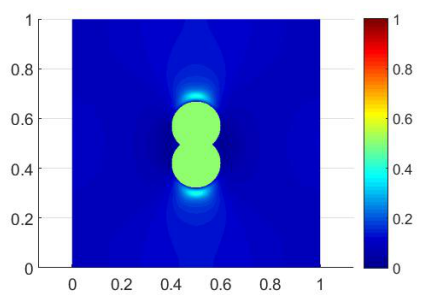

(a)

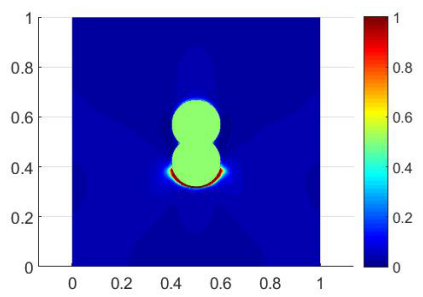

(d)

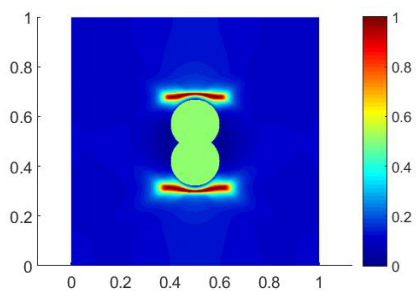

(b)

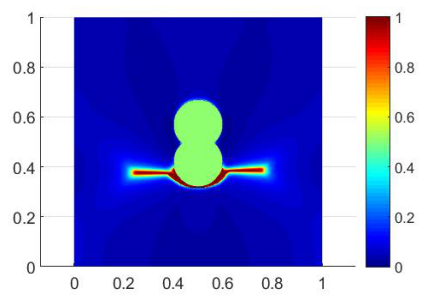

(e)

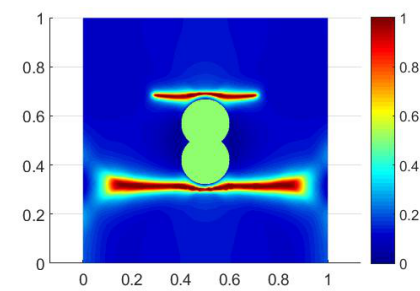

(c)

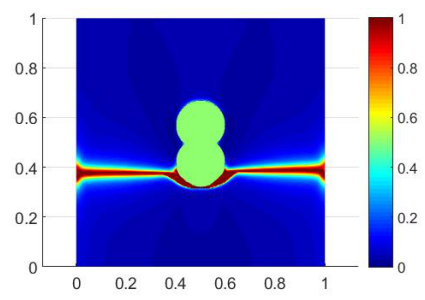

(f)

Fig. 25. Tensile test of a heterogeneous sample with complex inclusion: crack propagation. The phase field $d(\mathbf{x})$ is plotted. Figures (a), (b) and (c) depict the cracks initiation and propagation by using the classical phase field method and correspond to $\bar{U}=0.014 \mathrm{~mm}, \bar{U}=0.015 \mathrm{~mm}$, and $\bar{U}=0.016 \mathrm{~mm}$, respectively. Figs. (d), (e) and (f) depict crack propagation and initiation for the model including both phases and interfacial damage and correspond to $\bar{U}=0.008 \mathrm{~mm}, \bar{U}=0.01 \mathrm{~mm}$, and $\bar{U}=0.012 \mathrm{~mm}$, respectively.

The same boundary conditions, material parameters and loading increments as in the previous example are considered. Plane strain is assumed. A regular mesh of $400 \times 400$ elements is used. The regularization parameter $l$ is chosen as $l=0.005 \mathrm{~mm}$. We perform two simulations: one with the whole formulation, able to take into account both interfacial damage and matrix damage, and another which only takes into account damage of the bulk (basic phase field method). Damage evolution for each model is depicted in figure 25.

In this example and in all following ones, the domain does not contain preexisting cracks, and the cracks first nucleate and then propagate as the load 


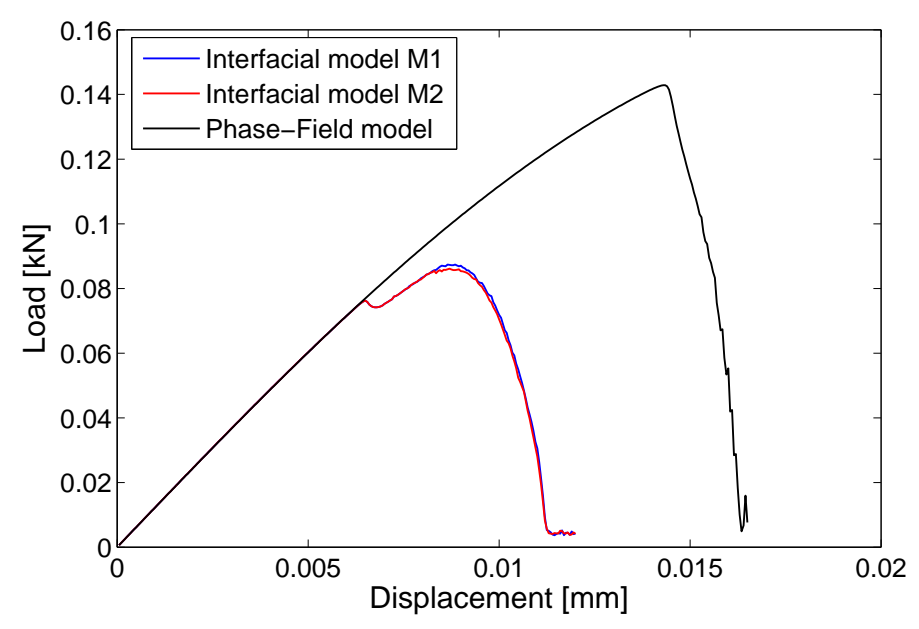

Fig. 26. Traction test of a complex microstructure: load-displacement curve.

is increased. We can note that in the case of the classical phase field method (damage only occurring in the phases), two cracks initiate on the top and at the bottom of the inclusion, where stress concentration occurs, and then propagate. In the case where interfacial damage is included, the damage clearly initiates in the interface and then propagates into the matrix. We note that in this case, only one crack propagates. The general dissymmetry is induced by the perturbation parameter $\epsilon$ (see Fig. 24 (a)) and due to the way the mesh cuts the exact disc and the assignment of material properties to the elements. The load-displacement curves are plotted for each case in Fig. 26.

We can note that both models M1 and M2 provide similar behaviors. The slight local minimum observed for the model with interfacial damage might be due to local relaxation associated to the damage near the interface. We can conclude that in the present framework, the use of additional internal variables (as in the M2 model) is unnecessary.

\subsection{Microcracking in a microstructure containing randomly distributed inclu- sions}

A microstructure containing 9 randomly distributed circular inclusions is considered, as depicted in Fig. 27 (a)-(b).

The microstructure is modeled as a square domain whose length is $L=1$ $\mathrm{mm}$. The radius of the inclusions is computed such that the volume fraction $f$ is equal to 0.07 . The elasticity parameters of each phase are the same as in the previous example: $E_{i}=52 \mathrm{GPa}, \nu_{i}=0.3, E_{m}=10.4 \mathrm{GPa}, \nu_{m}=$ 0.3 . The interfaces can be damaged through the model proposed in section 3.3.2, with again $t_{u}=10^{-2} \mathrm{GPa}$. The toughness of both phases is taken 


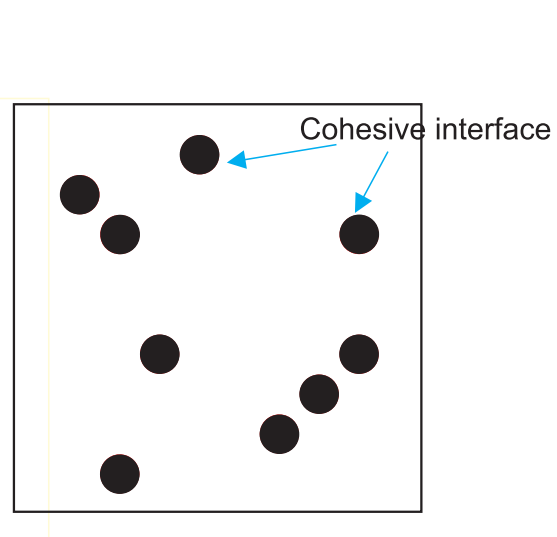

(a)

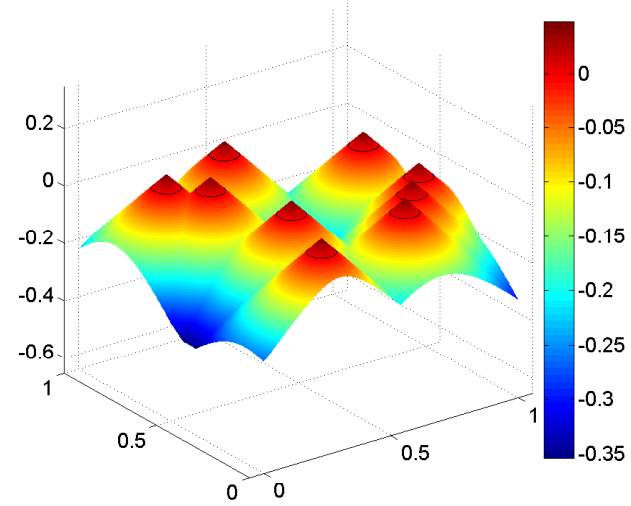

(c)

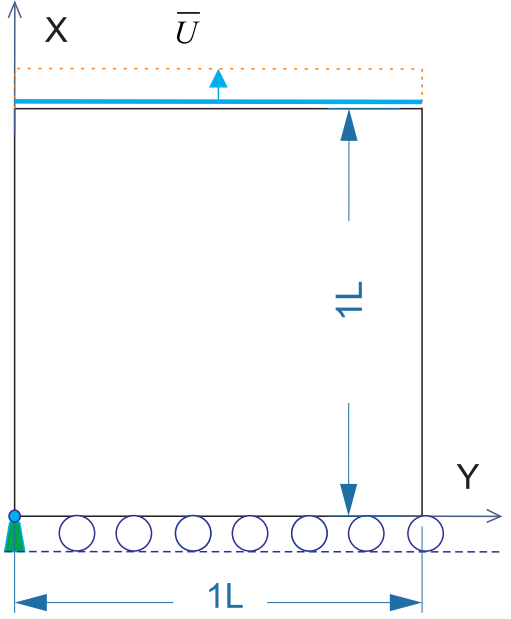

(b)

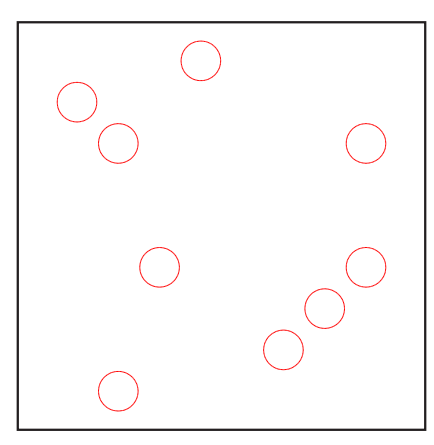

(d)

Fig. 27. Traction test of a microstructure containing randomly distributed inclusions: (a) geometry of the phases; (b) geometry of the domain and boundary conditions; (c) level-set function; (d) zero isocontour of the associated level-set

as $g_{c}=2.7 \times 10^{-4} \mathrm{kN} / \mathrm{mm}$ and $g_{c}^{I}=g_{c}$. The boundary conditions are as follows: on the lower end ( $y=0$, the $y$-displacements are fixed, while the $x$ displacements are free. The node at $(x=0),(y=0)$ is fixed. On the upper end, the $x$-displacements are free, while the $\mathrm{y}$-displacements are prescribed, with a uniform increasing value of $\bar{U}$ during the simulation. Lateral boundaries are free. Plane strain is assumed. The computation is performed with monotonic displacement increments of $\bar{U}=5 \times 10^{-5} \mathrm{~mm}$ during 200 increments. A regular mesh of $300 \times 300$ quadrilateral elements is used. The regularization parameter is chosen as $l=0.0075 \mathrm{~mm}$.

The microcracking distribution (damage variable $d(\mathbf{x})$ ) is depicted in Fig. 28 for several realizations of microstructures at a stage close to the peak stress.

In the different figures of Fig. 28, we can note that in each case, the cracks initiate at the interface between the matrix and the inclusions, and then propagate into the matrix. For most cases, a crack path is created between the inclusions 

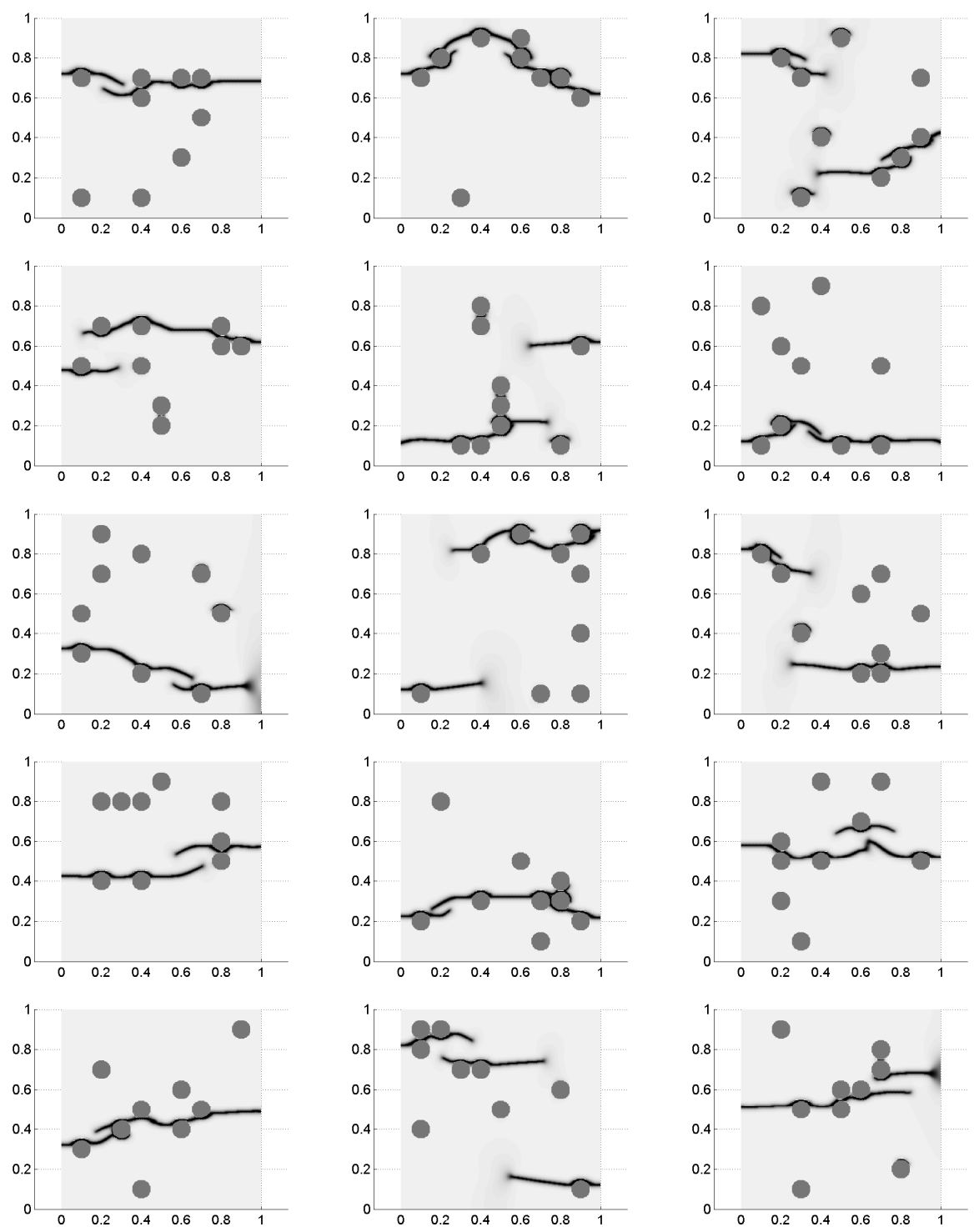

Fig. 28. Microcrocracking evolution for 15 realizations of microstructures in traction.

passing through the interfaces and then leading to the rupture of the specimen. This example illustrates well the capability and robustness of the technique to handle: (a) interaction between many cracks and (b) crack propagation from interfaces to the bulk. We note that the same regular mesh was used for each simulation, by only changing the level-set construction through the procedure described in section 4 .

The corresponding force-displacement curves are depicted in figure 29 for the set of 15 realizations. We note a relatively large dispersion of the individual results, with possibly non monotonous evolutions of the force/displacement curves. This shows on the one hand the strong sensitivity of the overall quasibrittle response to the local morphology of microstructure as well as the capability of the proposed method to capture these effect. This variability is 


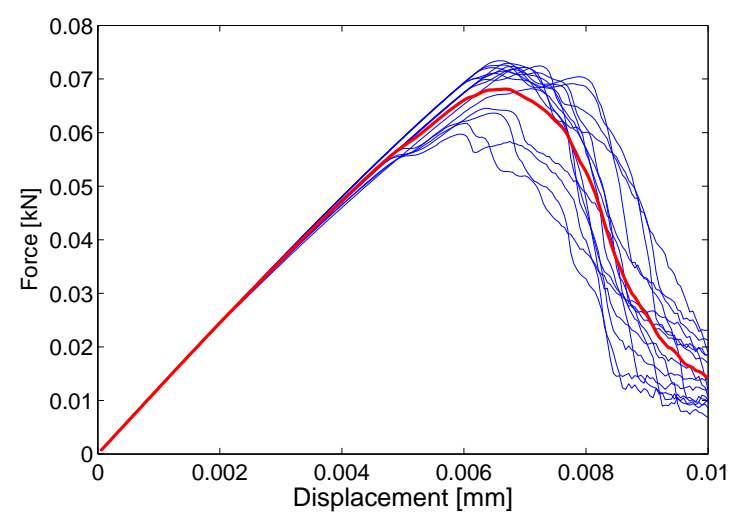

Fig. 29. Traction test of a microstructure containing randomly distributed inclusions with interfacial damage: load-deflection curve for 15 realizations. The red curve denotes the average response.

as expected much larger in the damaged part of the force displacement curve than in its initial linear part. On the other hand, the average of this individual responses, shown in red, exhibits a much more regular shape, which might be considered as a first evaluation of the overall softening response of a damaging composite containing much more inclusions. The convergence of individual simulations with increasing window sizes, or of their statistical averages, towards the effective behavior of such a composites remains a stringent open question. These preliminary results suggest that the proposed numerical tools might provide an efficient tool to make a big step for its investigation. More precisely, the concept of "representative volume element" for a quasi-brittle material might be clarified this way, following earlier ideas used for simpler linear elastic behaviours (e.g. [18]).

\subsection{Compression test of a realistic microstructure extracted from microto- mography image of an EPS lightweight concrete}

This last example illustrates the capability of the technique to solve problems of microcrack propagation in voxel-based models obtained from microtomography of real materials with both interfacial and bulk damage.

The considered 2D microstructure is a cross-section of a microtomography image of a real cementitious material obtained with the XRCT laboratory scanner available at Navier laboratory. The real material consists into quartz sand and almost spherical expanded polystyrene (EPS) beads with controlled diameter embedded into a cement matrix. The image was recorded for $1 \mathrm{~h} 20 \mathrm{~min}$ with a voxel size of $15 \mu \mathrm{m}$. The grey level image was filtered and thresholded to separate the three phases of the microstructure. The results are presented in Fig. 30 (a), where the white, grey and black phases correspond to matrix (cement paste), inclusions (sand grains) and pores (EPS beads), respectively. 


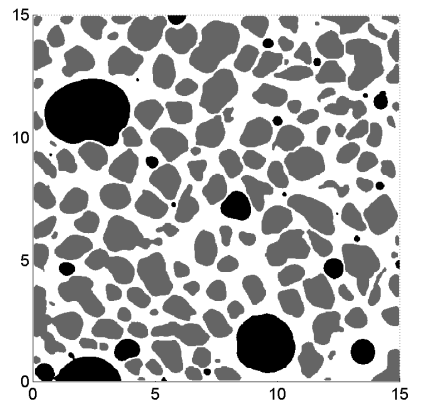

(a)

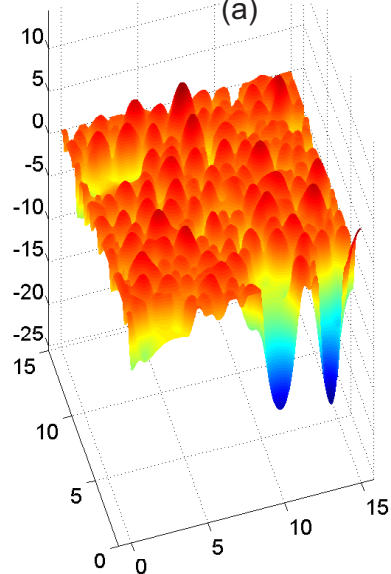

( c )

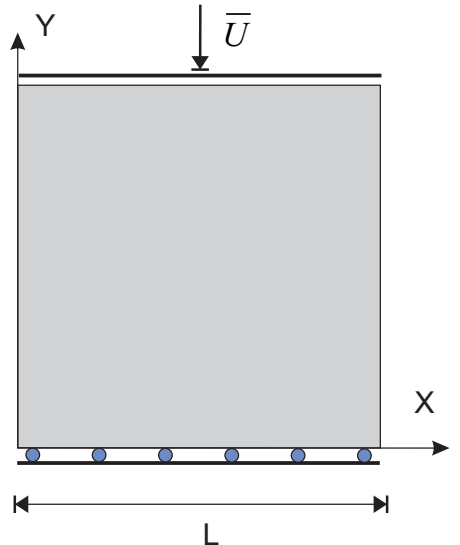

(b)

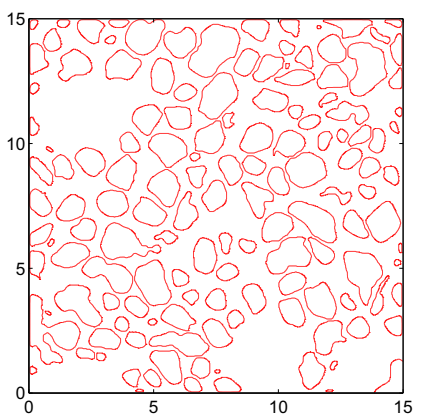

(d)

Fig. 30. Compression test of a microtomography image-based model of EPS lightweight concrete: (a) microstructure obtained from segmented image: the white, grey and black phases correspond to matrix, inclusions and pores, respectively; (b) geometry of the domain and boundary conditions; (c) level-set function and (d) zero isovalue of the constructed level-set.

The obtained level-set for this complex geometry described in section 4 is shown in Fig. 30 (c), and its zero isovalue, corresponding to the interface between the matrix and the rigid inclusions, is depicted in 30 (d). Note that the construction of a level-set function for such complex geometry is not trivial and proves the robustness of the original algorithm proposed in this work. Note that no level-set is used here to describe the matrix-pores interface, as it would make no sense to propagate cracks along such interfaces.

The boundary conditions and geometry of the sample are described in Fig. 30 (b) and are similar to the previous examples. From the $1000 \times 1000$ pixels, a mesh of $1000 \times 1000$ quadrilateral elements is generated, where the material property of matrix, inclusion or holes, are transferred into a regular grid of square domains associated with elements. The material parameters of inclusions and matrix are, respectively: $E_{i}=60 \mathrm{Gpa}, \nu_{i}=0.3, E_{m}=18 \mathrm{Gpa}$, and $\nu_{m}=0.2$. The pores are covered with elements of the regular mesh. We 


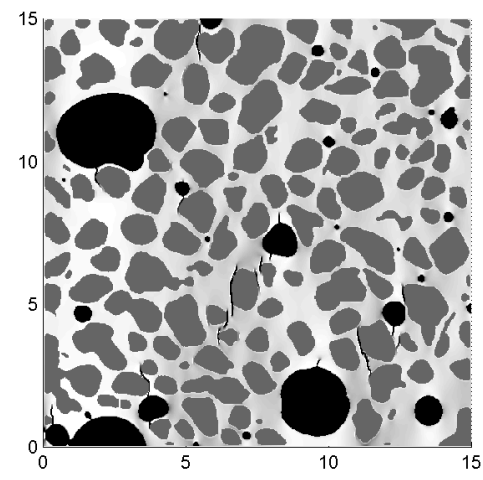

(a)

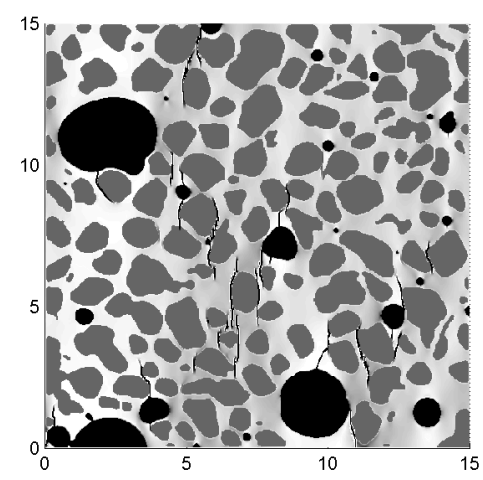

(c)

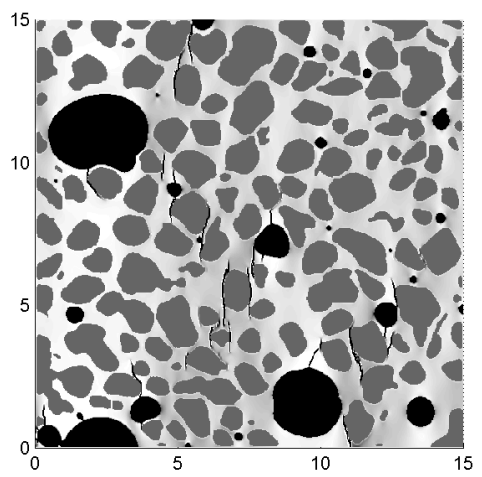

(b)

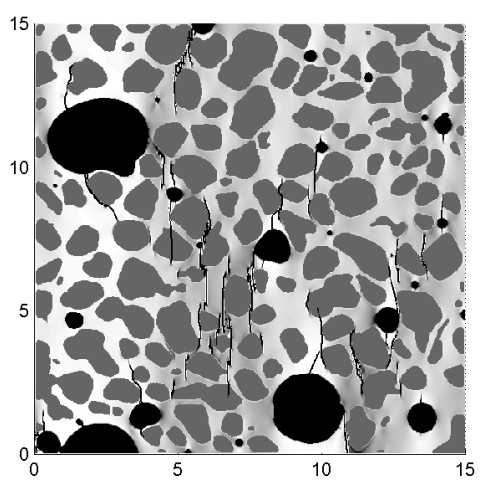

(d)

Fig. 31. Compression test on a microtomography image-based model of EPS lightweight concrete: microcracking evolution for: $u_{a}=0.12 \mathrm{~mm}, u_{b}=0.15 \mathrm{~mm}$, $u_{c}=0.162 \mathrm{~mm}$ and $u_{d}=0.18 \mathrm{~mm}$ (see Fig. 32 for corresponding force-displacement curve).

have chosen very compliant properties for these elements, $E_{p}=10^{-6} \mathrm{Gpa}$, $\nu_{p}=0$. The toughness $g_{c}=59.3 \mathrm{~N} / \mathrm{m}$ is assumed to be identical for the different phases and $g_{c}^{I}=g_{c}$. Note that in this example we have considered both normal and tangential components for the cohesive law, with $t_{u}=3.4$ MPa for both components [32]. The simulation is performed with monotonic displacement increments $\bar{U}=-6.10^{-4} \mathrm{~mm}$ during 250 load increments. The regularization parameter is chosen as $l=30 \mu \mathrm{m}$. The microcracking evolution in the domain is presented in Fig. 31.

We can observe several microcracks nucleation modes. In the vicinity of pores, cracks nucleate vertically, which is consistent with some recent experimental observations of plaster materials containing holes subjected to compression [31]. Other cracks nucleate from interfaces between inclusions and the matrix, and then propagate into the matrix. A few cracks propagate through inclusions. In all cases, the crack paths are very complex and show the potential of the method to describe microcracking with interfacial damage in very complex, 


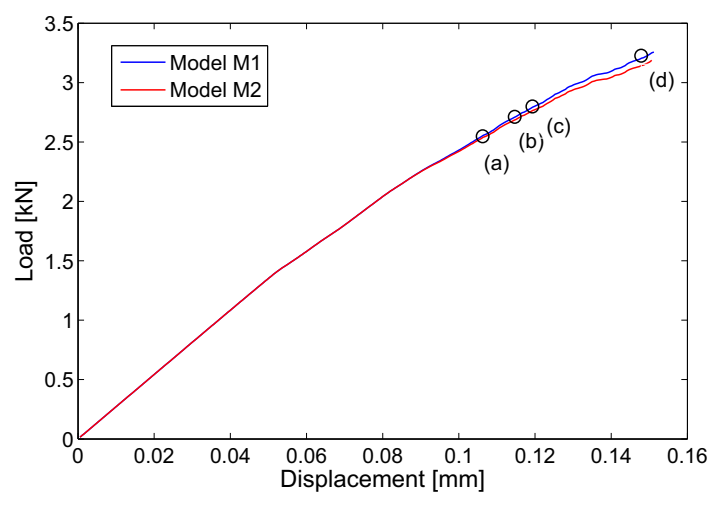

Fig. 32. Compression test of a microtomography image-based model of EPS lightweight concrete: load-deflection curve.

heterogeneous microstructures such as obtained by experimental imaging. The load-displacement curve is provided in Fig. 32. When microcracks start nucleating, the material strength quickly drops. We can note that both M1 and M2 models provide comparable solutions, which shows that the present framework can be employed with the simplest cohesive model and without internal interface variables, at least for monotonous loading conditions.

\section{Conclusion}

In this work, a phase field method capable of describing interactions between bulk brittle fracture and interfacial damage has been introduced. One main attractive feature of the phase field method is the diffuse approximation of discontinuities, which avoids the burden of remeshing during crack propagation and is well adapted to simulations of cracking within voxel-based models obtained for instance from X-ray CT images of real materials.

As compared to a previous contribution to phase field modeling of interfacial damage in [39], our new formulation allows describing opening and re-closure of cracks by using directly the phase field as an internal variable, which removes the need for internal variables related to cohesive interfaces. Then, the sole and unique phase field is employed to describe both bulk brittle fracture and interface cracking, and thus allows interaction between the two type of cracks. More specifically, we have simulated crack nucleation from interfaces and their propagation into the bulk.

To describe diffuse displacement jump at interfaces, a level-set method has been proposed, with a simple algorithm to construct the level-set function for arbitrary complex geometries arising from microtomography image-based models. 
The accuracy of the method has been proved through several well-known benchmarks (peeling test, crack propagation in 3-point bending of a beam). In addition, we have demonstrated the capability of the method to simulate interactions between interfacial damage and bulk brittle fractures for complex geometries arising from voxel-based model of microtomography images, which has been done for the first time in this work to our knowledge. The method then constitutes a very promising modeling and simulation tool for studying microcracking in a wide class of composite materials where both interfacial damage and matrix crack propagation occur, and could constitute a basis for many extensions of interfacial damage models involving other physical phenomena.

\section{Aknowledgements}

This work has benefited from a French government grant managed by ANR within the frame of the national program Investments for the Future ANR11-LABX-022-01. We thank prof. Karim Miled, professor at Ecole Nationale d'Ingénieurs de Tunis (ENIT), Tunisia, for providing us the cement paste samples used to obtain the microtomography image based models of porous microstructures. Finally, the financial support of Institut Universitaire de France (IUF) is acknowledged for J. Yvonnet.

\section{Appendix 1: analytical solution for the discontinuous benchmark problem}

In the following, we provide the exact solution of the discontinuous benchmark presented in example 6.1. The $u_{1}$ component of the displacement is linear in each domain, i.e.

$$
\begin{array}{ll}
u_{1}(x)=A_{1} x+B_{1} & \forall \mathbf{x} \in \Omega_{1}, \\
u_{1}(x)=A_{2} x+B_{2} & \forall \mathbf{x} \in \Omega_{1} .
\end{array}
$$


The constants are found by verifying the displacement boundary conditions and the continuity conditions at the interface $x=L / 2$ :

$$
\left\{\begin{array}{l}
u_{1}(x=0)=0 \\
u_{1}(x=L)=\bar{U} \\
\llbracket \sigma_{11}(x=L / 2) \rrbracket=0 \\
\sigma_{11}(x=L / 2)=t\left(\llbracket u_{1}(x=L / 2) \rrbracket\right)
\end{array}\right.
$$

which leads to the solution:

$$
\begin{aligned}
& u_{1}(x)=\frac{E_{2} \bar{U}}{\frac{L}{2}\left(E_{1}+E_{2}\right)+\frac{E_{1} E_{2} v_{n}^{2}}{g_{c}}} x \quad \text { for }\left(\mathrm{x}>\frac{\mathrm{L}}{2}\right) \\
& u_{1}(x)=\bar{U}-\frac{\bar{U}}{\frac{L}{2}\left(1+\frac{E_{2}}{E_{1}}\right)+\frac{E_{2} v_{n}^{2}}{g_{c}}}(L-x) \text { for }\left(\mathrm{x}<\frac{\mathrm{L}}{2}\right) .
\end{aligned}
$$

\section{Appendix 2: expressions of tangent components of the cohesive law}

The expressions of the terms in (54) are given by:

$$
\begin{aligned}
& D_{n n}=\frac{g_{c}^{I}}{\delta^{n}}\left(1-\frac{\mathrm{w}^{\mathrm{n}}}{\delta^{n}}\right) \exp \left(-\frac{\mathrm{w}^{\mathrm{n}}}{\delta^{\mathrm{n}}}\right) \exp \left(-\frac{\left(\mathrm{w}^{\mathrm{t}}\right)^{2}}{\left(\delta^{\mathrm{t}}\right)^{2}}\right), \\
& D_{n t}=-2 g_{c}^{I}\left(\frac{\mathrm{w}^{\mathrm{n}}}{\delta^{n}}\right)\left(\frac{\mathrm{w}^{\mathrm{t}}}{\left(\delta^{t}\right)^{2}}\right) \exp \left(-\frac{\mathrm{w}^{\mathrm{n}}}{\delta^{\mathrm{n}}}\right) \exp \left(-\frac{\left(\mathrm{w}^{\mathrm{t}}\right)^{2}}{\left(\delta^{\mathrm{t}}\right)^{2}}\right), \\
& D_{t t}=\frac{2 g_{c}^{I}}{\delta^{t}}\left(1-2 \frac{\left(w^{t}\right)^{2}}{\left(\delta^{t}\right)^{2}}\right)\left(1+\frac{\mathrm{w}^{\mathrm{n}}}{\delta^{n}}\right) \exp \left(-\frac{\mathrm{w}^{\mathrm{n}}}{\delta^{\mathrm{n}}}\right), \\
& D_{t n}=-2 g_{c}^{I}\left(\frac{\mathrm{w}^{\mathrm{t}}}{\delta^{t}}\right) \frac{\mathrm{w}^{\mathrm{n}}}{\left(\delta^{n}\right)^{2}} \exp \left(-\frac{\mathrm{w}^{\mathrm{n}}}{\delta^{\mathrm{n}}}\right) \exp \left(-\frac{\left(\mathrm{w}^{\mathrm{t}}\right)^{2}}{\left(\delta^{\mathrm{t}}\right)^{2}}\right) .
\end{aligned}
$$

\section{References}

[1] K. Akutagwa, K. Yamaguchi, A. Yamamoto, H. Heguri, H. Jinnai, and Y. Shinbari. Mesoscopic mechanical analysis of filled elastomers. Rubber Chem. Technol., 81(2):182-189, 2008. 
[2] L. Ambrosio and V.M. Tortorelli. Approximation of functional depending on jumps by elliptic functional via $\Gamma$-convergence. Commun. Pur. Appl. Math, 43(8):999-1036, 1990.

[3] H. Amor, J.J. Marigo, and C. Maurini. Regularized formulation of the variational brittle fracture with unilateral contact: Numerical experiments. $J$. Mech. Phys. Solids, 57(8):1209-1229, 2009.

[4] G.I. Barenblatt. The formation of equilibrium cracks during brittle fracture. general ideas and hypotheses. axially-symmetric cracks. J. Appl. Math. Mech., 23(3):622-636, 1959.

[5] P.E. Bernard, N. Moës, and N. Chevaugeon. Damage growth modeling using the thick level set (TLS) approach: Efficient discretization for quasi-static loadings. Comput. Meth. Appl. Mech. Eng., 233:11-27, 2012.

[6] M.J. Borden, T.J.R. Hughes, C.M. Landisb, and V. Verhooselc. A higherorder phase-field model for brittle fracture: Formulation and analysis within the isogeometric analysis framework. Comput. Meth. Appl. Mech., 273:100$118,2012$.

[7] D.P. Braides. Approximation of Free Discontinuity Problems. Springer Verlag, 1998.

[8] G.T. Camacho and M. Ortiz. Computational modelling of impact damage in brittle materials. Int. J. Solids. Struct, 33(20):2899-2938, 1996.

[9] F. Cazes and N. Moës. Comparison of a phase-field model and of a thick level set model for brittle and quasi-brittle fracture. Int. J. Numer. Methods Eng., In press, 2015.

[10] N. Chandra, H. Li, C. Shet, and H. Ghonem. Some issues in the application of cohesive zone models for metal-ceramic interfaces. Int. J. Solids. Struct, 39(10):2827-2855, 2002.

[11] Y. Chen, M. Pani, F. Taddei, and C. Mazzà. Large-scale finite element analysis of human cancellous bone tissue micro computer tomography data: a convergence study. J Biomech. Eng.-T. ASME, 136(10):101013, 2014.

[12] R. de Borst, J.J.C. Remmers, and C.V. Verhoosel. Evolving discontinuities and cohesive fracture. Procedia IUTAM, 10:125-137, 2014.

[13] M.J. Van den Bosch, P.J.G. Schreurs, and M.D.G Geers. An improved description of the exponential xu and needleman cohesive zone law for mixedmode decohesion. Eng. Fract. Mech, 73(9):1220-1234, 2006.

[14] D.S. Dugdale. Yielding of steel sheets containing slits. J. Mech. Phys. Solids, 8(2):100-104, 1960.

[15] G.A. Francfort and J.J. Marigo. Revisiting brittle fracture as an energy minimization problem. J. Mech. Phys. Solids, 46:1319-1342, 1998. 
[16] A. Godman and A. Bentur. Bond effects in high-strength silica fume concretes. ACI. Mater. J., 86(5), 1989.

[17] C. Jaeger. Rock mechanics and engineering. Cambridge University Press, 1979.

[18] T. Kanit, S. Forest, I. Galliet, V. Mounoury, and D. Jeulin. Determination of the size of the representative volume element for random composites: statistical and numerical approach. Int. J. Solids Struct., 40(13-14):3647 - 3679, 2003.

[19] K.M. Lee and O. Buyukozturk. Fracture mechanics parameters influencing the mechanical properties of high-performance concrete. ACI. Spe. P, 149, 1994.

[20] C. Miehe, M. Hofacker, and F. Welschinger. A phase field model for rateindependent crack propagation: Robust algorithmic implementation based on operator splits. Comput. Meth. Appl. Mech. Eng., 199:2776-2778, 2010.

[21] C. Miehe, F. Welschinger, and M. Hofacker. Thermodynamically consistent phase-field models of fracture: Variational principles and multi-field fe implementations. Int. J. Numer. Meth. Eng., 83:1273-1311, 2010.

[22] N. Moës, J. Dolbow, and T. Belytschko. A finite element method for crack growth without remeshing. Int. J. Numer. Meth. Eng, 46:131-150, 1999.

[23] D. Mumford and J. Shah. Optimal approximations by piecewise smooth functions and associated variational problems. Commun. Pur. Appl. Math, 42(5):577-685, 1989.

[24] J. Lamon N. Carrère, E. Martin. The influence of the interphase and associated interfaces on the deflection of matrix cracks in ceramic matrix composites. Compos. Part A, 31:1179-1190, 2000.

[25] A. Needleman. A continuum model for void nucleation by inclusion debonding. Journal of applied mechanics, 54(3):525-531, 1987.

[26] T.T. Nguyen, J. Yvonnet, K. Sab, M. Bornert, C. Chateau, R. Romani, and R. le Roy. On the choice of numerical parameters in the phase field method for simulating crack initiation with experimental validations. Submitted.

[27] T.T. Nguyen, J. Yvonnet, Q.-Z. Zhu, M. Bornert, and C. Chateau. A phase field method to simulate crack nucleation and propagation in strongly heterogeneous materials from direct imaging of their microstructure. Eng. Fract. Mech, 139:1839, 2014.

[28] M. Ortiz and A. Pandolfi. Finite-deformation irreversible cohesive elements for three-dimensional crack-propagation analysis. Int. J. Numer. Meth. Eng, 44(9):1267-1282, 1999.

[29] S. Osherand and N. Paragios. Geometric level set methods in imaging, vision, and graphics. Springer, 2003.

[30] W. Ren, Z. Yang, R. Sharma, C.H. Zhang, and P.J. Withers. Two-dimensional x-ray ct image based mesoscale fracture modelling of concrete. Eng. Fract. Mech., 133:24-39, 2015. 
[31] R. Romani, M. Bornert, D. Leguillon, R. Le Roy, and K. Sab. Detection of crack onset in double cleavage drilled specimens of plaster under compression by digital image correlation-theoretical predictions based on a coupled criterion. Eur. J. Mech. A-Solid, 51:172-182, 2015.

[32] J.G. Rots. Computational modeling of concrete fracture. PhD thesis, Technische Hogeschool Delft, 1988.

[33] L. Salvo, P. Cloetens, E. Maire, S. Zabler, J.J. Blandin, J. Y. Buffière, W. Ludwig, E. Boller, D. Bellet, and C. Josserond. X-ray micro-tomography: an attractive characterization technique in materials science. Nucl. Intrum. Methods, 200:273-286, 2003.

[34] C. Sandino, P. Kroliczek, D.D. McErlain, and S.K. Boyd. Predicting the permeability of trabecular bone by micro-computed tomography and finite element modeling. J. Biochem., 47:3129-3134, 2014.

[35] N. Sukumar, N. Moës, B. Moran, and T. Belytschko. Extended finite element method for three-dimensional crack modelling. Int. J. Numer. Meth. Eng, 48:1549-1570, 2000.

[36] N. Tsafnat, G. Tsafnat, and A.S. Jones. Micro-finite element modelling of oke blends using x-ray microtomography. Fuel, 87(13-14):2983-2987, 2008.

[37] V. Tvergaard. Model studies of fibre breakage and debonding in a metal reinforced by short fibres. J. Mech. Phys. Solids, 41:1309-1326, 1993.

[38] V. Tvergaard and J.W. Hutchinson. The influence of plasticity on mixed mode interface toughness. J. Mech. Phys. Solids, 41(6):1119-1135, 1993.

[39] C.V. Verhoosel and R. de Borst. A phase-field model for cohesive fracture. Int. J. Numer. Meth. Eng, 96(1):43-62, 2013.

[40] Y.S. Wang, G.Y. Huang, and D. Gross. On the mechanical modeling of functionally graded interfacial zone with a griffith crack: plane deformation. Int. J. Fracture, 125(1):189-205, 2004.

[41] G.N. Wells and L.J. Sluys. A new method for modelling cohesive cracks using finite elements. Int. J. Numer. Meth. Eng, 50(12):2667-2682, 2001.

[42] X.P. Xu and A. Needleman. Numerical simulations of fast crack growth in brittle solids. J. Mech. Phys. Solids, 42(9):1397-1434, 1994.

[43] J. Yvonnet. A fast method for solving microstructural problems defined by digital images: a Space Lippmann-Schwinger scheme. Int. J. Numer. Meth. Eng., 92(2):178-205, 2012.

[44] F. Zhou and J.F. Molinari. Dynamic crack propagation with cohesive elements: a methodology to address mesh dependency. Int. J. Numer. Meth. Eng., 59:124, 2004. 